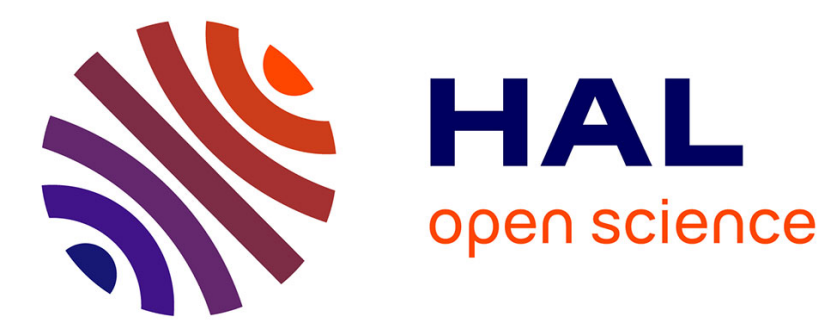

\title{
High-Fidelity Aerostructural Gradient Computation Techniques with Application to a Realistic Wing Sizing
}

Timothée Achard, Christophe Blondeau, Roger Ohayon

\section{To cite this version:}

Timothée Achard, Christophe Blondeau, Roger Ohayon. High-Fidelity Aerostructural Gradient Computation Techniques with Application to a Realistic Wing Sizing. AIAA Journal, 2018, 56 (11), pp.4487-4499. 10.2514/1.J056736 . hal-02462349

\section{HAL Id: hal-02462349 \\ https://hal.science/hal-02462349}

Submitted on 31 Jan 2020

HAL is a multi-disciplinary open access archive for the deposit and dissemination of scientific research documents, whether they are published or not. The documents may come from teaching and research institutions in France or abroad, or from public or private research centers.
L'archive ouverte pluridisciplinaire HAL, est destinée au dépôt et à la diffusion de documents scientifiques de niveau recherche, publiés ou non, émanant des établissements d'enseignement et de recherche français ou étrangers, des laboratoires publics ou privés. 


\title{
High-Fidelity Aerostructural Gradient Computation
}

\section{Techniques with Application to a Realistic Wing}

\author{
Sizing \\ Timothée Achard ${ }^{1}$ and Christophe Blondeau ${ }^{2}$ \\ ONERA - The French Aerospace Lab, F-92322 Châtillon, France \\ Roger Ohayon $^{3}$ \\ CNAM, Paris, F-75003, France
}

Aerostructural optimization is a keystone process to concurrently improve aerodynamic performance and reduce the structural mass of an aircraft. However, gradientbased multidisciplinary design optimization is efficient only if the computation of gradients is fast and accurate. To this end, we propose two high-fidelity aerostructural gradient computation techniques for strongly coupled aeroelastic systems. In the specific context of this work, we focus on design variables affecting structural stiffness only. Scalar objective functions like aerodynamic performance criteria are considered, as well as a field of structural grid forces. The most intrusive technique includes wellestablished direct and adjoint formulations that require substantial implementation effort. In contrast, we propose an alternative uncoupled non-intrusive approach easier to implement and yet capable of providing accurate gradient approximations. The accuracy of these methods is first demonstrated on the ONERA M6 Wing test-case. Their efficiency and applicability are then illustrated via a mass minimization problem applied to the Common Research Model (CRM). Both methods lead to very similar optimal designs and the detailed analysis of results promotes the non-intrusive approach as a promising gradient computation alternative.

\footnotetext{
1 PhD candidate, Aerodynamics, Aeroelasticity and Acoustics Department, 29 Avenue de la Division Leclerc.

2 Research Scientist, Aerodynamics, Aeroelasticity and Acoustics Department, 29 Avenue de la Division Leclerc.

3 Professor, Structural Mechanics and Coupled Systems Laboratory, 2 rue Conté, AIAA Fellow.
} 


\section{Nomenclature}

$\mathbf{X}_{a} \quad=$ aerodynamic grid

$\mathbf{X}_{s} \quad=$ structural grid

$\mathbf{X}_{a 0}=$ reference aerodynamic grid

$J \quad=$ function of interest

$\mathbf{p}=$ structural design variables

$\mathbf{W}=$ fluid conservative variables

$\mathbf{U}=$ structural displacements

$\mathbf{R}_{a}=$ discrete aerodynamic residuals

$\mathbf{R}_{s}=$ discrete structural residuals

$\mathbf{Q}_{a}=$ aerodynamic loads applied on the fluid grids belonging to the aeroelastic interface

$\mathbf{Q}_{s}=$ structural loads applied on the structural grids belonging to the aeroelastic interface

$\mathbf{T}_{\text {surf }}^{Q}=$ linear load transfer operator

$\mathbf{T}_{v o l}=$ volumic operator performing the deformation of the fluid interior domain

$\delta \mathbf{X}_{\text {surf }}^{a}=$ displacements of the fluid nodes at the aeroelastic interface

$\mathbf{T}_{\text {surf }}^{U}=$ linear displacement transfer operator

$\boldsymbol{\Lambda}_{a}=$ aerodynamic adjoint vector

$\boldsymbol{\Lambda}_{s} \quad=$ structural adjoint vector

$\mathbf{\Phi} \quad=$ structural eigenvectors

$\mathbf{q}=$ generalized coordinates

$\gamma \quad=$ generalized stiffness matrix

$n_{\Phi} \quad=$ number of retained eigensolutions

$n_{p} \quad=$ number of structural design parameters

$\mathbf{U}_{\text {res }}=$ residual structural displacements

$\mathbf{G A F}=$ generalized aerodynamic forces matrix

$\mathbf{S} \quad=$ flexibility matrix

TFI = Transfinite Interpolation

IDW $=$ Inverse Distance Weighting 


\section{Introduction}

To improve the aircraft efficiency, one can either do incremental improvements over standard aircraft design (typically pylon fairing or winglet redesign from the A320 to the A320 neo) and obtain safely some marginal improvements at each design step or go along a more risky path for disruptive new configurations and get potentially higher improvements. However, in both cases a way to get better or radical new designs relies on the aerostructural optimization of the aircraft to find the proper trades between aerodynamic and structure disciplines.

Structural optimization with respect to sizing and shape parameters was developed during the late 1960s (see review by Adelman and Haftka [1]). Aerodynamic optimization started in the 1970s, essentially for subsonic flows, using low-fidelity models [2]. Aeroelastic optimization, defined as the consideration of fluid-structure interactions during the aerodynamic optimization, appeared in the late 1980s [3]. In the same period, the forward swept composite wing box design of the Grumman X-29 marked the beginning of a new kind of structural sizing. Indeed, the need for a proper optimization of the composite wing box structure was dictated by the static divergence instability inherent to the forward-swept wing configuration. This instability was prevented by using composite materials instead of metal alloys and by tailoring the composite stacking sequence in order to generate a favorable aeroelastic interaction. This process lies under the well-known discipline of aeroelastic tailoring [4].

When composite materials are used or more generally for large aspect ratio wings, the performance of an aircraft naturally depends more heavily on the fluid-structure interactions. In this case, the use of an aerostructural design optimization, that is the concurrent optimization of aerodynamic and structure taking into account fluid-structure interactions, is recommended to capture all the physics involved in the system. However, these interactions have been usually neglected due to time constraints in the design cycle. Indeed, taking into account these interactions significantly increases the computational time. This led designers to follow a sequential optimization process, with each discipline gaining maturity separately. Unfortunately, this strategy can lead to a non-optimal multidisciplinary design. Consequently, a strong coupling between the different disciplines in a multidisciplinary optimization loop is a key factor to identify sooner and in a reliable way the best 
potential configurations in the early design stage. For instance in 1988, Grossman et al. [5] showed that the sequential aerostructural optimization applied on a sailplane wing was clearly less effective than the integrated one (i.e. with a strong coupling). However, the counterpart of an integrated gradient-based optimization process is the added difficulty associated to cross-sensitivities computation. These cross-terms are typically the derivatives of aerodynamic performance with respect to structural sizes and the derivatives of structural response with respect to changes in aerodynamic shape [5]. Similar conclusions were drawn by Chittick and Martins [6].

The simplest way to compute gradients is the finite differences method. It has been used regularly since the beginning of numerical optimization (see $[7,8]$ ), and is still used today, for instance in the Jacobian Free Newton Krylov (JFNK) method to compute Jacobian-vector products $[9,10]$. However, this method has several issues concerning its accuracy and its cost. The value of the gradient obtained can be very sensitive to the amplitude of the design perturbation, requiring a fine tuning of the step size [11]. Furthermore, the cost of the method is directly indexed on the number of design variables. Another easy gradient computation technique is the complex-step method [12]. This method is robust with respect to numerical truncation, which is a great advantage in terms of accuracy. However, the code should be able to handle complex arithmetic which is a strong requirement.

Sobieszczanski-Sobieski proposed in 1990 [13] a computational framework to obtain the gradients of coupled systems. It is a direct analytic method, based on the linearization of the functions solution of the coupled system, leading to the so-called Global Sensitivity Equations. This gradient computation method is independent of the number of responses, but dependent on the number of design variables. It was applied by Maute et al. in 2001 [14] in an aeroelastic optimization methodology for an aerostructural system, using high-fidelity CFD/CSM models.

For an aerodynamic optimization, there are usually few responses, but many design variables. It is then preferable to compute gradients using the adjoint method, whose computational cost is indexed to the number of responses and is quasi-independent of the number of design variables. In 1988, Jameson [15] introduced the adjoint method for aerodynamic shape optimization purposes. It has been followed by numerous research works on aerodynamic shape optimization using the 
adjoint method, with a particular focus on increasing the fidelity of the models [16-21]. These research works were based on manual implementation of the adjoint solver.

Hand-differentiating a code is a tedious and non-trivial task, in particular when the objective is to obtain a coupled gradient computation capability. This is why some authors proposed the concept of Automatic Differentiation (AD) [22]. This method is attractive due to its consistency (the derivatives obtained are very close to the exact derivatives of the numerical model), and its apparent simplicity. However, the source code is often partly differentiable only, and the resulting code generated is modular but might be hardly readable. Nevertheless, it is a promising way which is subject to active research [23]. For instance, it was used in 2012 by Kenway et al. [24] for developing an aerostructural gradient computation module in combination with analytic differentiation of the code. More recently, Sanchez et al. [25] proposed a general approach that applies AD to a full partitioned fluid-structure solver, and implemented this solution in the open-source SU2 software.

At ONERA, active research work dedicated to aerostructural optimization was conducted during the past decade. In 2008, Marcelet [26] developed a module capable of computing static aeroelastic equilibrium and sensitivity analysis using the adjoint method. However, the structural model behavior was limited to equivalent beam model kinematics, and the aeroelastic gradients were only computed with respect to aerodynamic shape parameters. In a follow-on work, Ghazlane extended this work to take into account structural parameters (typically stiffener cross-section, plate thickness...) for aerostructural gradients computation [27]. Lastly, Viti [28] demonstrated a bi-step aerostructural preliminary design of a forward-swept wing applying these gradient computation tools. Nonetheless, the structural model was still limited to a beam model in all these works. Although useful for preliminary design studies and design space exploration, this structural modeling is not able to tackle complex aeroelastic couplings, nor deal with composite structures for efficient aeroelastic tailoring design, and is obviously limited to high aspect ratio wings. Meanwhile, several developments have been made over the last decade to compute high-fidelity aerostructural gradients using adjoint method $[24,29,30]$.

The objective of a previous work [31] by the authors was to relieve all these limitations by re-developing an aerostructural gradient capability from scratch, by systematic hand-differentiation 
of discretized aeroelastic equations, and all associated operators, in the els $\boldsymbol{A}$ aeroelasticity module (elsA/Ael) [32]. This way, the new capability inherits the structural paradigm embedded into the els $\boldsymbol{A} /$ Ael module, the whole catalog of fluid-structure transfer methods, as well as the underlying parallel architecture. The equations and the solving procedure for the aeroelastic and the aerostructural gradients have been discussed in [31], where only aeroelastic shape gradients computation capability (i.e. gradients of aeroelastic functions of interest with respect to aerodynamic shape

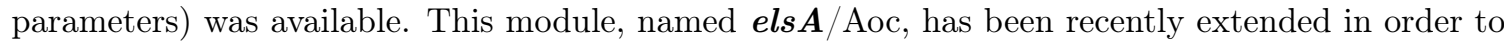
take into account structural design parameters in the direct and the adjoint mode. This extension is covered by the present paper.

The efficiency of the adjoint approach holds when a reduced number of responses is considered compared to a large set of design parameters. However, an industrial wing box sizing for preliminary design considers up to several hundreds of design parameters and up to several tens of thousands of structural constraints. In order to still benefit from the elegant adjoint formulation, some authors have used a constraint aggregation approach $[33,34]$ as an attempt to reduce the size of the set of admissible constraints, thus the number of gradients to compute. However, this technique has several drawbacks. Typically, the approximation quality of the classical Kreisselmeier-Steinhauser (KS) aggregation function is controlled by an aggregation parameter $\rho$ which has to be properly tuned. If many constraints are active at the optimum, this aggregation technique may lead to suboptimal designs $[35,36]$. Nevertheless, recent research has shown that the use of new constraint aggregation classes in conjunction with well-established practices could be efficient for structural sizing [37].

Considering these difficulties, the authors have proposed in a previous paper [38] a new strategy to compute high-fidelity gradients of aerodynamic functions of interest with respect to structural parameters. This strategy is based on an uncoupled non-intrusive approach benefiting from the existing linearized aerodynamic module dedicated to gradient computation. The main advantage of the method is the independence of the computational cost with respect to the number of constraints and potentially with respect to the number of structural design parameters. In addition this approach only requires rigid CFD computations. This new gradient computation method may be used 
in conjunction with the Force Approximation Method [39] to approximate the loads rather than the design responses. Indeed, the loads are easier to approximate due to their smoother distribution. Finally, the use of constraint screening, which only retains the salient constraints in the optimization problem (violated, active, almost active) reduces further the computational cost.

The objective of this work is to compare the efficiency of the non-intrusive approach with the classical intrusive direct or adjoint method for aerostructural gradient computation. Both approaches are investigated on the ONERA M6 Wing (see [40]) and the Common Research Model (CRM) [41] test-cases. Criteria such as accuracy, efficiency and applicability on practical industrial problems are discussed.

The remainder of this paper is organized as follows: Section II details the intrusive direct and adjoint approaches. Section III presents an improved version of the non-intrusive method originally proposed in [38]. The gradient results are shown in Sec. IV where the various approaches are compared and discussed on the M6 Wing test-case. Finally, we consider in Sec. V a mass minimization of the CRM wing box under stress constraints.

\section{Intrusive approach}

Let's assume that a steady aeroelastic equilibrium has been previously computed. In the following, we will denote the corresponding mesh as "flight shape", as opposed to the unloaded reference mesh called "jig shape". This is illustrated in Fig. 1, where $\mathbf{X}_{a}$ is the equilibrium aerodynamic mesh, $\mathbf{X}_{s}$ the structural mesh, and $\mathbf{X}_{a 0}$ the aerodynamic reference mesh. Our objective is to compute the sensitivity of a function of interest $J$ with respect to a set of structural design variables p affecting the stiffness matrix only. To this end, direct and adjoint approaches derived from the linearization of the aeroelastic equations around the equilibrium position will be used.

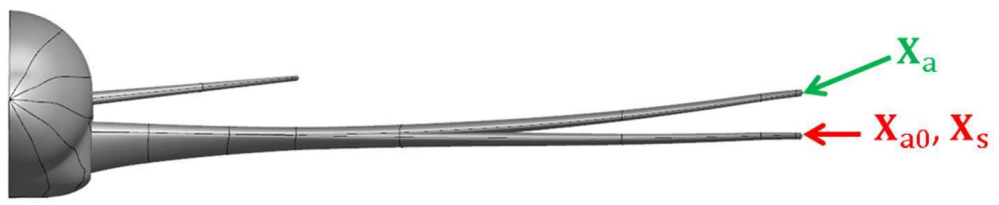

Flight shape defining the fluid and structure states Reference aerodynamic and structure grids

Fig. 1 Definition of the different meshes. 
Let's denote the state variables of the coupled system $\mathbf{W}$ and $\mathbf{U}$, representing the fluid conservative variables and the structural displacements. At the aeroelastic equilibrium, the state variables and the meshes satisfy the discretized equations of fluid and structural mechanics simultaneously:

$$
\left\{\begin{array}{l}
\mathbf{R}_{a}\left(\mathbf{X}_{a}, \mathbf{W}, \mathbf{U}\right)=\mathbf{0} \\
\mathbf{R}_{s}\left(\mathbf{X}_{s}, \mathbf{W}, \mathbf{U}\right)=\mathbf{0}
\end{array}\right.
$$

where $\mathbf{R}_{a}$ is the discrete aerodynamic residual and $\mathbf{R}_{s}$ the discrete structure residual, forming respectively a nonlinear and a linear system of equations. These two blocks of equations are coupled through aerodynamic forces $\mathbf{Q}_{a}$ loading the structure skin and the structural displacements $\mathbf{U}$ deforming the fluid mesh. The structural loads $\mathbf{Q}_{s}$ are obtained with a suitable load transfer technique applied to $\mathbf{Q}_{a}$ :

$$
\mathbf{Q}_{s}\left(\mathbf{Q}_{a}\left(\mathbf{W}, \mathbf{X}_{a}\right), \mathbf{X}_{a 0}, \mathbf{X}_{s}\right)=\mathbf{T}_{\text {surf }}^{Q}\left(\mathbf{X}_{a 0}, \mathbf{X}_{s}\right) \mathbf{Q}_{a}\left(\mathbf{W}, \mathbf{X}_{a}\right)
$$

where $\mathbf{T}_{\text {surf }}^{Q}\left(\mathbf{X}_{a 0}, \mathbf{X}_{s}\right)$ represents a linear load transfer operator. The structural displacements alter the fluid grid positions through the relation:

$$
\mathbf{X}_{a}=\mathbf{X}_{a 0}+\delta \mathbf{X}_{a}\left(\delta \mathbf{X}_{a, \text { surf }}, \mathbf{X}_{a 0}\right)=\mathbf{X}_{a 0}+\mathbf{T}_{\text {vol }}\left(\mathbf{X}_{a 0}\right) \delta \mathbf{X}_{a, \text { surf }}
$$

with $\mathbf{T}_{v o l}\left(\mathbf{X}_{a 0}\right)$ the volume operator performing the deformation of the fluid interior domain. The mesh deformation is a "one shot" technique based on a combination of an Inverse Distance Weighting (IDW) step for fluid block boundaries followed by a linear TransFinite Interpolation (TFI) for the fluid blocks interior. The vector $\delta \mathbf{X}_{a, s u r f}$ corresponds to the displacements of the fluid nodes at the aeroelastic interface:

$$
\delta \mathbf{X}_{a, \text { surf }}=\delta \mathbf{X}_{a, \text { surf }}\left(\mathbf{X}_{a 0}, \mathbf{X}_{s}, \mathbf{U}\right)=\mathbf{T}_{\text {surf }}^{U}\left(\mathbf{X}_{a 0}, \mathbf{X}_{s}\right) \mathbf{U}
$$

where $\mathbf{T}_{\text {surf }}^{U}\left(\mathbf{X}_{a 0}, \mathbf{X}_{s}\right)$ represents a linear displacement transfer operator.

\section{A. Direct approach}

Let's consider the scalar aeroelastic objective function $J\left(\mathbf{W}, \mathbf{X}_{a}, \mathbf{X}_{s}\right)$ and a structural parameter p. We assume that the implicit function theorem is applicable to our problem. In this case, the state variables $\mathbf{W}$ and $\mathbf{U}$ can be expressed as implicit functions $\mathbf{W}(p)$ and $\mathbf{U}(p)$ of the optimization 
parameters and have the same regularity properties as $\mathbf{R}_{a}$ and $\mathbf{R}_{s}$. Direct differentiation of system 1 with respect to $p$ yields the tangent problem for the aerostructural gradient:

$$
\left[\begin{array}{ll}
\frac{\partial \mathbf{R}_{a}}{\partial \mathbf{W}} & \frac{\partial \mathbf{R}_{a}}{\partial \mathbf{U}} \\
\frac{\partial \mathbf{R}_{s}}{\partial \mathbf{W}} & \frac{\partial \mathbf{R}_{s}}{\partial \mathbf{U}}
\end{array}\right]\left[\begin{array}{l}
\frac{d \mathbf{W}}{d p} \\
\frac{d \mathbf{U}}{d p}
\end{array}\right]=\left[\begin{array}{c}
-\frac{\partial \mathbf{R}_{a}}{\partial \mathbf{X}_{a}} \frac{d \mathbf{X}_{a}}{d p} \\
-\frac{\partial \mathbf{R}_{s}}{\partial \mathbf{X}_{s}} \frac{d \mathbf{X}_{s}}{d p}-\frac{\partial \mathbf{R}_{s}}{\partial p}
\end{array}\right]
$$

The system above has been simplified since there is no explicit dependency of the aerodynamic residual $\mathbf{R}_{a}$ with respect to $p$. Neglecting the partial derivative $\partial J / \partial p$, the total gradient of the objective function $J$ is then written as:

$$
\frac{d J}{d p}=\frac{\partial J}{\partial \mathbf{W}} \frac{d \mathbf{W}}{d p}+\frac{\partial J}{\partial \mathbf{X}_{a}} \frac{d \mathbf{X}_{a}}{d p}+\frac{\partial J}{\partial \mathbf{X}_{s}} \frac{d \mathbf{X}_{s}}{d p}
$$

Our objective is now to expand Eq. 5 and 6 with respect to the unknown vectors $d \mathbf{W} / d p$ and $d \mathbf{U} / d p$. First, we recall that $p$ affects only the stiffness of the structure, so that the following relations hold:

$$
\frac{\partial \mathbf{X}_{a 0}}{\partial p}=\frac{\partial \mathbf{X}_{s}}{\partial p}=\mathbf{0}
$$

We then differentiate Eq. 3 with respect to $p$, and using Eq. 4, we get:

$$
\frac{d \mathbf{X}_{a}}{d p}=\frac{d \delta \mathbf{X}_{a}}{d p}=\mathbf{T}_{\text {vol }} \mathbf{T}_{\text {surf }}^{U} \frac{d \mathbf{U}}{d p}
$$

Substituting these two equations into Eq. 6 yields the expression of the total gradient of the objective function:

$$
\frac{d J}{d p}=\frac{\partial J}{\partial \mathbf{W}} \frac{d \mathbf{W}}{d p}+\frac{\partial J}{\partial \mathbf{X}_{a}} \mathbf{T}_{\text {vol }} \mathbf{T}_{\text {surf }}^{U} \frac{d \mathbf{U}}{d p}
$$

Terms $\partial J / \partial \mathbf{X}_{a}$ and $\partial J / \partial \mathbf{W}$ only depend on the equilibrium steady state and are calculated analytically in a pre-processing step.

Let's rewrite the second block of the system in Eq. 5 taking into account Eq. 7:

$$
\frac{\partial \mathbf{R}_{s}}{\partial \mathbf{U}} \frac{d \mathbf{U}}{d p}+\frac{\partial \mathbf{R}_{s}}{\partial \mathbf{W}} \frac{d \mathbf{W}}{d p}=-\frac{\partial \mathbf{R}_{s}}{\partial p}
$$

The structural residual can be written as $\mathbf{R}_{s}(p)=\mathbf{K}(p) \mathbf{U}(p)-\mathbf{Q}_{s}(p)$, where $\mathbf{K}$ is the stiffness matrix, and $\mathbf{Q}_{s}$ the structural loads. First recognizing that:

$$
\frac{\partial \mathbf{R}_{s}}{\partial \mathbf{U}}=\mathbf{K}-\frac{\partial \mathbf{Q}_{s}}{\partial \mathbf{U}}=\mathbf{K}-\mathbf{T}_{\text {surf }}^{Q} \frac{\partial \mathbf{Q}_{a}}{\partial \mathbf{U}}
$$


and using the functional dependency of the structural loads (see Eq. 2):

$$
\frac{\partial \mathbf{Q}_{a}}{\partial \mathbf{U}} \frac{d \mathbf{U}}{d p}=\frac{\partial \mathbf{Q}_{a}}{\partial \mathbf{W}_{b}} \frac{\partial \mathbf{W}_{b}}{\partial \mathbf{X}_{a, \text { surf }}} \frac{d \mathbf{X}_{a, \text { surf }}}{d p}+\frac{\partial \mathbf{Q}_{a}}{\partial \mathbf{X}_{a, \text { surf }}} \frac{d \mathbf{X}_{a, \text { surf }}}{d p}
$$

where $\mathbf{W}_{b}$ are the conservative variables at the boundary (aerodynamic skin), and $\mathbf{X}_{a, \text { surf }}$ is the corresponding aerodynamic surface mesh. The partial derivative of the structural residual with respect to the fluid states reads:

$$
\frac{\partial \mathbf{R}_{s}}{\partial \mathbf{W}}=-\frac{\partial \mathbf{Q}_{s}}{\partial \mathbf{W}}=-\mathbf{T}_{\text {surf }}^{Q} \frac{\partial \mathbf{Q}_{a}}{\partial \mathbf{W}_{b}} \frac{\partial \mathbf{W}_{b}}{\partial \mathbf{W}}
$$

Using the relation $\partial \mathbf{R}_{s} / \partial p=(\partial \mathbf{K} / \partial p) \mathbf{U}$, and from Eq. 8 the relation $d \mathbf{X}_{a, s u r f} / d p=\mathbf{T}_{\text {surf }}^{U}(d \mathbf{U} / d p)$, the structural block finally takes the following form:

$$
\mathbf{K} \frac{d \mathbf{U}}{d p}=\mathbf{T}_{\text {surf }}^{Q}\left[\frac{\partial \mathbf{Q}_{a}}{\partial \mathbf{W}_{b}}\left(\frac{\partial \mathbf{W}_{b}}{\partial \mathbf{W}} \frac{d \mathbf{W}}{d p}+\frac{\partial \mathbf{W}_{b}}{\partial \mathbf{X}_{a, \text { surf }}} \mathbf{T}_{\text {surf }}^{U} \frac{d \mathbf{U}}{d \mathbf{p}}\right)+\frac{\partial \mathbf{Q}_{a}}{\partial \mathbf{X}_{a, s u r f}} \mathbf{T}_{\text {surf }}^{U} \frac{d \mathbf{U}}{d \mathbf{p}}\right]-\frac{\partial \mathbf{K}}{\partial p} \mathbf{U}
$$

In the expression above, all the partial derivatives are computed analytically. $\partial \mathbf{W}_{b} / \partial \mathbf{W}$ and $\partial \mathbf{W}_{b} / \partial \mathbf{X}_{a, s u r f}$ are obtained by linearizing the boundary conditions (typically wallslip in Euler and no-slip in RANS) with respect to the conservative variables and the metric, respectively. It should be noted that the no-slip condition simplifies the expression above. Indeed, $\partial \mathbf{W}_{b} / \partial \mathbf{X}_{a, \text { surf }}$ is null in this case due to the flow attachment boundary condition. Similarly, $\partial \mathbf{Q}_{a} / \partial \mathbf{W}_{b}$ and $\partial \mathbf{Q}_{a} / \partial \mathbf{X}_{a, \text { surf }}$ are obtained by linearizing the fluxes at the aerodynamic skin with respect to the conservative variables and the metric, respectively.

Using relation 14, system 5 can be cast into the compact form:

$$
\left[\begin{array}{cc}
\frac{\partial \mathbf{R}_{a}}{\partial \mathbf{W}} & \frac{\partial \mathbf{R}_{a}}{\partial \mathbf{X}_{a}}[\mathbf{A}] \\
-[\mathbf{B}] & \mathbf{K}-[\mathbf{C}]
\end{array}\right]\left[\begin{array}{c}
\frac{d \mathbf{W}}{d p} \\
\frac{d \mathbf{U}}{d p}
\end{array}\right]=\left[\begin{array}{c}
\mathbf{0} \\
-\frac{\partial \mathbf{K}}{\partial p} \mathbf{U}
\end{array}\right]
$$

Constant matrices $[\mathbf{A}],[\mathbf{B}]$ and $[\mathbf{C}]$ are defined analytically with the following formulas

$$
\begin{aligned}
& {[\mathbf{A}]=\mathbf{T}_{\text {vol }} \mathbf{T}_{\text {surf }}^{U}} \\
& {[\mathbf{B}]=\mathbf{T}_{\text {surf }}^{Q} \frac{\partial \mathbf{Q}_{a}}{\partial \mathbf{W}}} \\
& {[\mathbf{C}]=\mathbf{T}_{\text {surf }}^{Q} \frac{\partial \mathbf{Q}_{a}}{\partial \mathbf{X}_{a, \text { surf }}} \mathbf{T}_{\text {surf }}^{U}}
\end{aligned}
$$

Following the same notations, Eq. 9 becomes:

$$
\frac{d J}{d p}=\frac{\partial J}{\partial \mathbf{W}} \frac{d \mathbf{W}}{d p}+\frac{\partial J}{\partial \mathbf{X}_{a}}[\mathbf{A}] \frac{d \mathbf{U}}{d p}
$$


The coupled system in Eq. 15 is solved with an iterative block scheme similar to the laggedblock strategy proposed in [14]. If superscript $k$ refers to the current iteration number, Algorithm 1 details the Linear Block Gauss Seidel (LBGS) scheme used for the direct approach. The relaxation parameter $\theta$ has been introduced on the gradient of the structural displacements $d \mathbf{U} / d p$ to improve convergence of the coupled system solution.

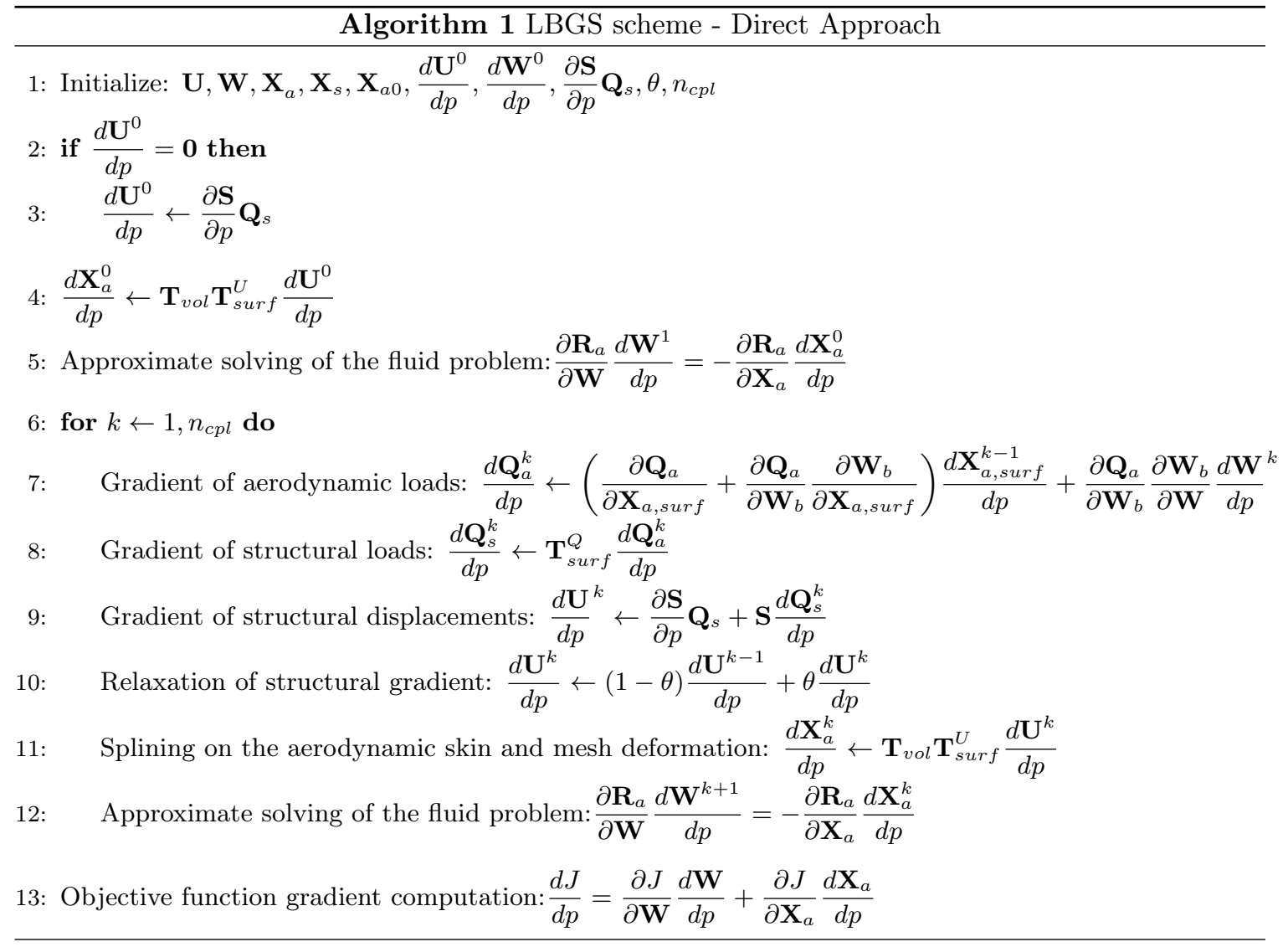

In Algorithm 1, the flexibility matrix $\mathbf{S}$ has been introduced. This matrix is obtained during a pre-processing step on the Finite Element Model (FEM). Using this matrix, the structural residual $\mathbf{R}_{s}=\mathbf{K}(p) \mathbf{U}(p)-\mathbf{Q}_{s}(p)$ actually reads $\mathbf{R}_{s}=\mathbf{U}(p)-\mathbf{S}(p) \mathbf{Q}_{s}(p)$. $\mathbf{S}$ is not to be confused with the inverse of the full stiffness matrix. It is much like a transfer function which relates a set of structural forces applied at selected nodes to a set of displacements on the aeroelastic interface. In practice, the set of force nodes is designed to ensure a realistic load path into the structure (input to $\mathbf{T}_{\text {surf }}^{Q}$ ) and the set of displacement nodes contains the grids input to the displacement transfer operator $\mathbf{T}_{\text {surf }}^{U}$. Thus, the size of $\mathbf{S}$ is small compared to the number of degrees of freedom in the FEM. 


\section{B. Adjoint approach}

In order to obtain the adjoint equations of the aerostructural gradient, the objective function is formulated by adding the total variation of the residuals $\mathbf{R}_{a}$ and $\mathbf{R}_{s}$ to $d J / d p$. These variations with respect to the design variables being null, we can write for any vector $\boldsymbol{\Lambda}_{a}$ and $\boldsymbol{\Lambda}_{s}$ :

$$
\frac{d J}{d p}=\frac{\partial J}{\partial \mathbf{W}} \frac{d \mathbf{W}}{d p}+\frac{\partial J}{\partial \mathbf{X}_{a}}[\mathbf{A}] \frac{d \mathbf{U}}{d p}+\boldsymbol{\Lambda}_{a}^{T} \frac{d \mathbf{R}_{a}}{d p}+\boldsymbol{\Lambda}_{s}^{T} \frac{d \mathbf{R}_{s}}{d p}
$$

Expanding $d \mathbf{R}_{a} / d p$ and $d \mathbf{R}_{s} / d p$ and factoring out the difficult terms $d \mathbf{W} / d p$ and $d \mathbf{U} / d p$ yields:

$$
\begin{aligned}
\frac{d J}{d p} & =\left(\frac{\partial J}{\partial \mathbf{W}}+\boldsymbol{\Lambda}_{a}^{T} \frac{\partial \mathbf{R}_{a}}{\partial \mathbf{W}}-\boldsymbol{\Lambda}_{s}^{T}[\mathbf{B}]\right) \frac{d \mathbf{W}}{d p} \\
& +\left(\frac{\partial J}{\partial \mathbf{X}_{a}}[\mathbf{A}]+\boldsymbol{\Lambda}_{a}^{T} \frac{\partial \mathbf{R}_{a}}{\partial \mathbf{X}_{a}}[\mathbf{A}]+\boldsymbol{\Lambda}_{s}^{T}(\mathbf{K}-[\mathbf{C}])\right) \frac{d \mathbf{U}}{d p}+\boldsymbol{\Lambda}_{s}^{T} \frac{\partial \mathbf{K}}{\partial p} \mathbf{U}
\end{aligned}
$$

where adjoint vectors $\boldsymbol{\Lambda}_{a}$ and $\boldsymbol{\Lambda}_{s}$ are chosen such that the following system is satisfied:

$$
\left[\begin{array}{cc}
{\left[\frac{\partial \mathbf{R}_{a}}{\partial \mathbf{W}}\right]^{T}} & -[\mathbf{B}]^{T} \\
{[\mathbf{A}]^{T}\left[\frac{\partial \mathbf{R}_{a}}{\partial \mathbf{X}_{a}}\right]^{T}} & \mathbf{K}^{T}-[\mathbf{C}]^{T}
\end{array}\right]\left[\begin{array}{l}
\boldsymbol{\Lambda}_{a} \\
\boldsymbol{\Lambda}_{s}
\end{array}\right]=\left[\begin{array}{c}
-\left[\frac{\partial J}{\partial \mathbf{W}}\right]^{T} \\
-[\mathbf{A}]^{T}\left[\frac{\partial J}{\partial \mathbf{X}_{a}}\right]^{T}
\end{array}\right]
$$

Finally, once adjoint unknown vectors are determined, the total gradient is obtained with:

$$
\frac{d J}{d p}=\boldsymbol{\Lambda}_{s}^{T} \frac{\partial \mathbf{K}}{\partial p} \mathbf{U}
$$

Practically, the adjoint system is solved using the flexibility matrix $\mathbf{S}$ rather than the stiffness matrix K. In this case, system 20 reads:

$$
\left[\begin{array}{cc}
{\left[\frac{\partial \mathbf{R}_{a}}{\partial \mathbf{W}}\right]^{T}} & -[\mathbf{B}]^{T} \mathbf{S}^{T} \\
{[\mathbf{A}]^{T}\left[\frac{\partial \mathbf{R}_{a}}{\partial \mathbf{X}_{a}}\right]^{T}} & \mathbf{I}-[\mathbf{C}]^{T} \mathbf{S}^{T}
\end{array}\right]\left[\begin{array}{c}
\boldsymbol{\Lambda}_{a} \\
\boldsymbol{\Lambda}_{s}
\end{array}\right]=\left[\begin{array}{c}
-\left[\frac{\partial J}{\partial \mathbf{W}}\right]^{T} \\
-[\mathbf{A}]^{T}\left[\frac{\partial J}{\partial \mathbf{X}_{a}}\right]^{T}
\end{array}\right]
$$

and the total gradient of the objective function writes:

$$
\frac{d J}{d p}=-\boldsymbol{\Lambda}_{s}^{T} \frac{\partial \mathbf{S}}{\partial p} \mathbf{Q}_{s}
$$

The process for solving the adjoint system follows an iterative block scheme analogous to that used for the direct system in Eq. 15. The relaxation factors $\theta_{a}$ and $\theta_{s}$ have been introduced on the Lagrange multipliers $\boldsymbol{\Lambda}_{a}$ and $\boldsymbol{\Lambda}_{s}$. Algorithm 2 details the Linear Block Gauss Seidel (LBGS) scheme used for the adjoint approach. 


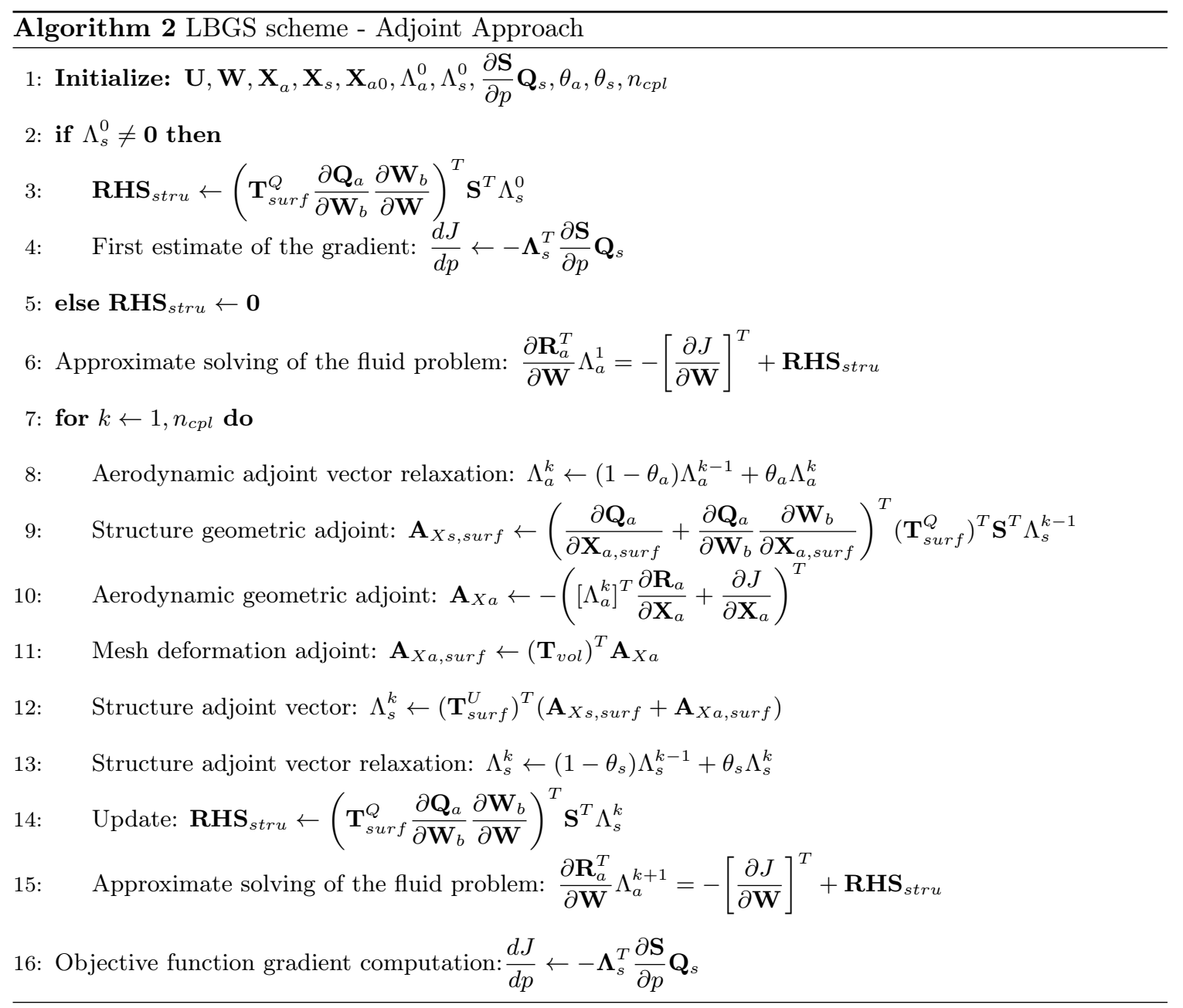

Direct and adjoint equations have been written here for the particular case of a scalar aeroelastic function of interest (lift or drag coefficient), with optimization parameters controlling only the stiffness of the structure (e.g. stiffener cross-section, plate thickness). In a previous work by the authors [31], these equations have been detailed for the more general case of parameters affecting both the shape and stiffness sizes. The adjoint system of Eq. 20 is independent of the nature of the design parameters. Only the objective function gradient assembly differs. This constitutes a major advantage in the simultaneous optimization of both aerodynamic and structure.

\section{Non-intrusive approach}

The non-intrusive technique allows a complete decoupling of the structure and aerodynamic analyses. The idea consists in building a projection of the static displacement field on the structural modeshapes in order to re-use the linearized kernel of the flow solver optimization module. 
This approach has been discussed in [38]. With this strategy, aerostructural gradients have been computed on the ONERA M6 wing and then on the CRM configuration with satisfactory results. In this section, we first establish the link between gradients of loads and gradients of structural displacements through the linearized solver. Then, the equations leading to the modal reconstruction of the aeroelastic load sensitivities are presented.

\section{A. Working process with the linearized solver}

Let's consider the tangent system in Eq. 15. Solving the first block of equations and substituting $d \mathbf{W} / d p$ in the structure block yields:

$$
\left\{\begin{array}{l}
(\mathbf{K}-[\mathbf{Z}]) \frac{d \mathbf{U}}{d p}=-\frac{\partial \mathbf{K}}{\partial p} \mathbf{U} \\
{[\mathbf{Z}]=-[\mathbf{B}]\left[\frac{\partial \mathbf{R}_{a}}{\partial \mathbf{W}}\right]^{-1} \frac{\partial \mathbf{R}_{a}}{\partial \mathbf{X}_{a}}[\mathbf{A}]+[\mathbf{C}]}
\end{array}\right.
$$

Matrix $(\mathbf{K}-[\mathbf{Z}])$ appearing in the first block of equations is the Schur complement of the matrix block $\partial \mathbf{R}_{a} / \partial \mathbf{W}$. We recall that direct differentiation of the static equilibrium equation $\mathbf{K U}=\mathbf{Q}_{s}$ with respect to $p$ gives:

$$
\frac{\partial \mathbf{K}}{\partial p} \mathbf{U}+\mathbf{K} \frac{d \mathbf{U}}{d p}=\frac{d \mathbf{Q}_{s}}{d p}
$$

Comparing this expression with the first block of equation in system 24 yields the relation:

$$
\frac{d \mathbf{Q}_{s}}{d p}=[\mathbf{Z}] \frac{d \mathbf{U}}{d p}
$$

where the linear operator $[\mathbf{Z}]$ embeds the kernel matrix of the linearized flow solver, which links the gradients of structural displacements to the gradients of structural loads.

\section{B. Modal reconstruction of aerostructural gradients from an aeroelastic configuration}

The steady equilibrium corresponding to a fluid state $\mathbf{W}$, a mesh position $\mathbf{X}_{a}$ and a structural displacement field $\mathbf{U}$ takes the following form:

$$
\mathbf{K U}=\mathbf{Q}_{s}=\left[\mathbf{T}_{\text {surf }}^{Q}\right] \mathbf{Q}_{a}\left(\mathbf{W}, \mathbf{X}_{a}\right)
$$

where $\mathbf{K}$ is the stiffness matrix of the finite element model, $\mathbf{Q}_{s}$ the nodal structural loads, and $\mathbf{Q}_{a}$ the aerodynamic loads computed on the aerodynamic skin. If the full set $\boldsymbol{\Phi}$ of structural eigenvectors 
was available, the modal projection $\mathbf{U}=\mathbf{\Phi q}$ would hold exactly. Then substituting in Eq. 27 gives the corresponding generalized coordinates as:

$$
\mathbf{q}=\gamma^{-1} \boldsymbol{\Phi}^{T} \mathbf{Q}_{s}
$$

where $\gamma=\boldsymbol{\Phi}^{T} \mathbf{K} \boldsymbol{\Phi}$ denotes the diagonal generalized stiffness matrix. In practice only a restricted set of first $n_{\boldsymbol{\Phi}}$ eigensolutions is computed and the modal approximation to $\mathbf{U}$ becomes:

$$
\mathbf{U} \approx \mathbf{U}_{\Phi}=\sum_{i=1}^{n_{\Phi}} \boldsymbol{\Phi}_{i} q_{i}
$$

However, recall that $\mathbf{U}$ is computed exactly from Eq. 27 such that the residual term in Eq. 29 is known from the simple difference $\mathbf{U}_{r e s}=\mathbf{U}-\mathbf{U}_{\Phi}$. Equation 27 can now be reformulated as

$$
\mathbf{K}\left(\mathbf{U}_{\Phi}+\mathbf{U}_{r e s}\right)=\mathbf{Q}_{s}
$$

Inserting the modal decomposition of $\mathbf{U}$ in the expression of the gradient of structural loads (Eq. 26) leads to

$$
\frac{d \mathbf{Q}_{s}}{d p}=\mathbf{Z} \boldsymbol{\Phi} \frac{d \mathbf{q}}{d p}+\mathbf{Z}\left(\frac{\partial \boldsymbol{\Phi}}{\partial p} \mathbf{q}+\frac{\partial \mathbf{U}_{r e s}}{\partial p}\right)
$$

The use of partial derivatives in the equation above means that $\boldsymbol{\Phi}$ and $\mathbf{U}_{r e s}$ only depend on $p$. The latter assumption is valid if $\mathbf{U}_{r e s}$ is considered as a static residual mode (i.e. a structural displacement under an assumed prescribed load case). In order to exploit Eq. 31, the gradient of the generalized coordinates has to be determined. With some algebraic manipulation, it is possible to show that:

$$
\frac{d \mathbf{q}}{d p}=[\boldsymbol{\gamma}-\mathbf{G A F}]^{-1}\left(-\frac{\partial \boldsymbol{\gamma}}{\partial p} \mathbf{q}+\boldsymbol{\Phi}^{T} \mathbf{Z}\left(\frac{\partial \boldsymbol{\Phi}}{\partial p} \mathbf{q}+\frac{\partial \mathbf{U}_{r e s}}{\partial p}\right)\right)
$$

where the matrix of generalized aerodynamic forces is defined as $\mathbf{G A F}=\boldsymbol{\Phi}^{T} \mathbf{Z} \mathbf{\Phi}$.

The first part $(\mathbf{Z} \boldsymbol{\Phi})$ of Eq. 31 costs one linearized computation per mode. The second part $\left(\mathbf{Z}\left[(\partial \boldsymbol{\Phi} / \partial p) \mathbf{q}+\partial \mathbf{U}_{r e s} / \partial p\right]\right)$ requires one linearized computation per parameter. Once these two parts are determined, the gradient of the generalized coordinates is readily available.

Usually, few mode shapes are necessary to correctly approximate the structural displacements (Eq. 29). However, many design variables can be defined in the optimization problem. In this case, and in its current form, the method is no longer of interest since it is independent of the number 
of objective functions, but dependent on the number of design variables. In an attempt to reduce the computational cost, gradients can be approximated by dropping the terms which scale with the number of design variables. In this case Eq. 32 and 31 become:

$$
\begin{gathered}
\frac{d \mathbf{q}}{d p}=[\boldsymbol{\gamma}-\mathbf{G A F}]^{-1}\left(-\frac{\partial \boldsymbol{\gamma}}{\partial p} \mathbf{q}\right) \\
\frac{d \mathbf{Q}_{s}}{d p}=\mathbf{Z} \boldsymbol{\Phi} \frac{d \mathbf{q}}{d p}
\end{gathered}
$$

\section{Extension to shape optimization}

Initially the non-intrusive approach was intended to be used in a bi-level MDO formulation [42] and to allow efficient structural sizing under high-fidelity CFD loads at the structure discipline level. This is why in its current formulation and implementation it is devoted to structural design parameters only. However, this approach can easily be extended to shape design parameters provided that additional partial derivatives like $\partial \mathbf{X}_{a 0} / \partial p$ for the aerodynamic contribution and $\partial \mathbf{K} / \partial p, \partial \mathbf{\Phi} / \partial p$ and $\partial \gamma / \partial p$ for the structural contributions are provided by the user. We recall that $\mathbf{X}_{a 0}$ is the reference aerodynamic grid (see Fig. 1). Although the structural grid coordinates also depend on the shape design parameters, the sensitivity $\partial \mathbf{X}_{s} / \partial p$ does not appear explicitely in the equations. In this case, the coupled system in Eq. 15 exhibits additional contributions to its right-hand side, associated to the aerodynamic grid parameterization:

$$
\left[\begin{array}{cc}
\frac{\partial \mathbf{R}_{a}}{\partial \mathbf{W}} & \frac{\partial \mathbf{R}_{a}}{\partial \mathbf{X}_{a}}[\mathbf{A}] \\
-[\mathbf{B}] & \mathbf{K}-[\mathbf{C}]
\end{array}\right]\left[\begin{array}{c}
\frac{d \mathbf{W}}{d p} \\
\frac{d \mathbf{U}}{d p}
\end{array}\right]=\left[\begin{array}{c}
-\frac{\partial \mathbf{R}_{a}}{\partial \mathbf{X}_{a}} \mathbf{T}_{\text {vol }} \frac{\partial \mathbf{X}_{a 0, \text { surf }}}{\partial p} \\
-\frac{\partial \mathbf{K}}{\partial p} \mathbf{U}+\mathbf{T}_{\text {surf }}^{Q} \frac{\partial \mathbf{Q}_{a}}{\partial \mathbf{X}_{a, \text { surf }}} \frac{\partial \mathbf{X}_{a 0, \text { surf }}}{\partial p}
\end{array}\right]
$$

The expression in Eq. 26 for the total derivative of structural loads is updated accordingly to give:

$$
\frac{d \mathbf{Q}_{s}}{d p}=[\mathbf{Z}]\left(\mathbf{T}_{\text {surf }}^{U} \frac{\partial \mathbf{U}}{\partial p}+\frac{\partial \mathbf{X}_{a 0, \text { surf }}}{\partial p}\right)
$$

where a new linear mapping $[\mathbf{Z}]$ has been defined as:

$$
[\mathbf{Z}]=-[\mathbf{B}]\left[\frac{\partial \mathbf{R}_{a}}{\partial \mathbf{W}}\right]^{-1} \frac{\partial \mathbf{R}_{a}}{\partial \mathbf{X}_{a}} \mathbf{T}_{\text {vol }}+\mathbf{T}_{\text {surf }}^{Q} \frac{\partial \mathbf{Q}_{a}}{\partial \mathbf{X}_{a, s u r f}}
$$


Finally, the sensitivity of the generalized coordinates becomes:

$$
\frac{d \mathbf{q}}{d p}=[\boldsymbol{\gamma}-\mathbf{G} \mathbf{A F}]^{-1}\left(-\frac{\partial \boldsymbol{\gamma}}{\partial p} \mathbf{q}+\boldsymbol{\Phi}^{T}[\mathbf{Z}]\left(\mathbf{T}_{\text {surf }}^{U}\left(\frac{\partial \mathbf{\Phi}}{\partial p} \mathbf{q}+\frac{\partial \mathbf{U}_{r e s}}{\partial p}\right)+\frac{\partial \mathbf{X}_{a 0, \text { surf }}}{\partial p}\right)\right)
$$

where the new matrix of generalized aerodynamic forces is defined as: GAF $=\boldsymbol{\Phi}^{T}[\mathbf{Z}] \mathbf{T}_{\text {surf }}^{U} \boldsymbol{\Phi}$. It is important to note that the computational cost does not change compared to the previous formulation as the number of calls to the linearized aerodynamic solver remains identical. Therefore the non-intrusive aproach can be equally effective in a full aerostructural optimization.

\section{M6 Wing test-case}

In this section, we apply the intrusive and non-intrusive approaches to the ONERA M6 wing. Our objective is to compute lift and drag coefficient sensitivities with respect to structural parameters, as well as gradients of structural loads. As detailed in subsection V A, these latter can be used to deduce the gradients of structural responses (such as displacements, strains and stresses) with respect to structural design variables, for structural sizing purpose.

The fluid computational structured Euler mesh depicted in Fig. 2 (left) contains 1.11 million cells divided into five blocks. The Mach number for this case is $\mathrm{M}=0.734$ and the incidence is $2.8^{\circ}$. The wing tip displacement is $6.57 \mathrm{~cm}$ (i.e. $5.48 \%$ of span) and the associated flexible lift coefficient is $\mathrm{Cz}=0.195$. An upwind Roe scheme with a MUSCL interpolation associated to a Van Albada limiting function is applied.

The finite element model presented in Fig. 2 (right) has a classical wing box layout with spars, ribs and stiffeners. Member thicknesses and sections have been designed in a pre-processing optimization step. This model can be easily tuned in order to control flexibility and consequently aeroelastic effects. Two design parameters have been defined for the structural model. First parameter $p_{1}$ controls the thickness of the upper and lower skins, and second parameter $p_{2}$ affects the thickness of the three spar webs.

For the non-intrusive approach, the first six structural mode shapes have been retained in the structural displacement approximation. These mode shapes are smoothed on the aerodynamic skin and plotted in Fig. 3 below.

For the intrusive approach, the convergence of the discrete residual norm of the fluid block is 

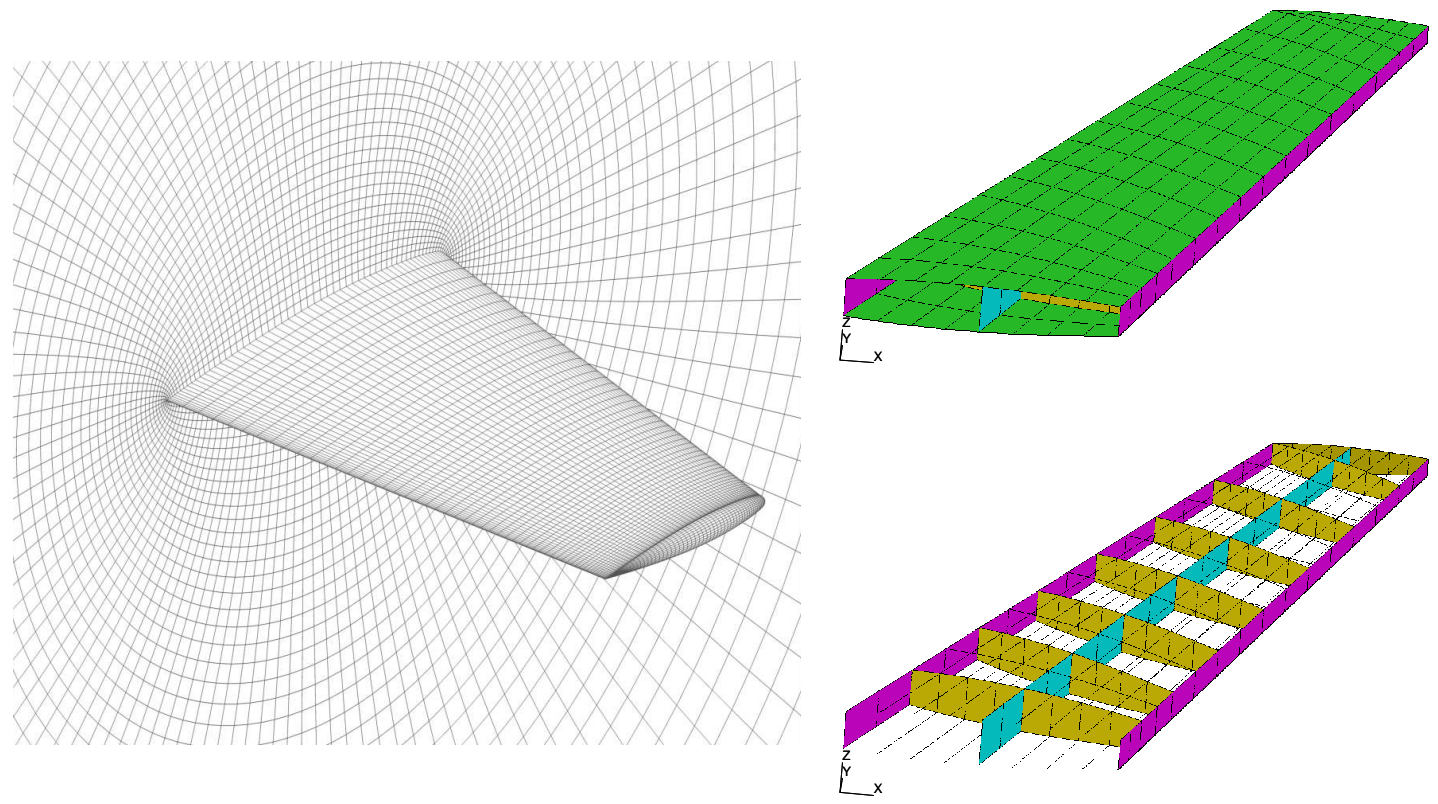

Fig. 2 M6 wing Euler fluid mesh and structural mesh.

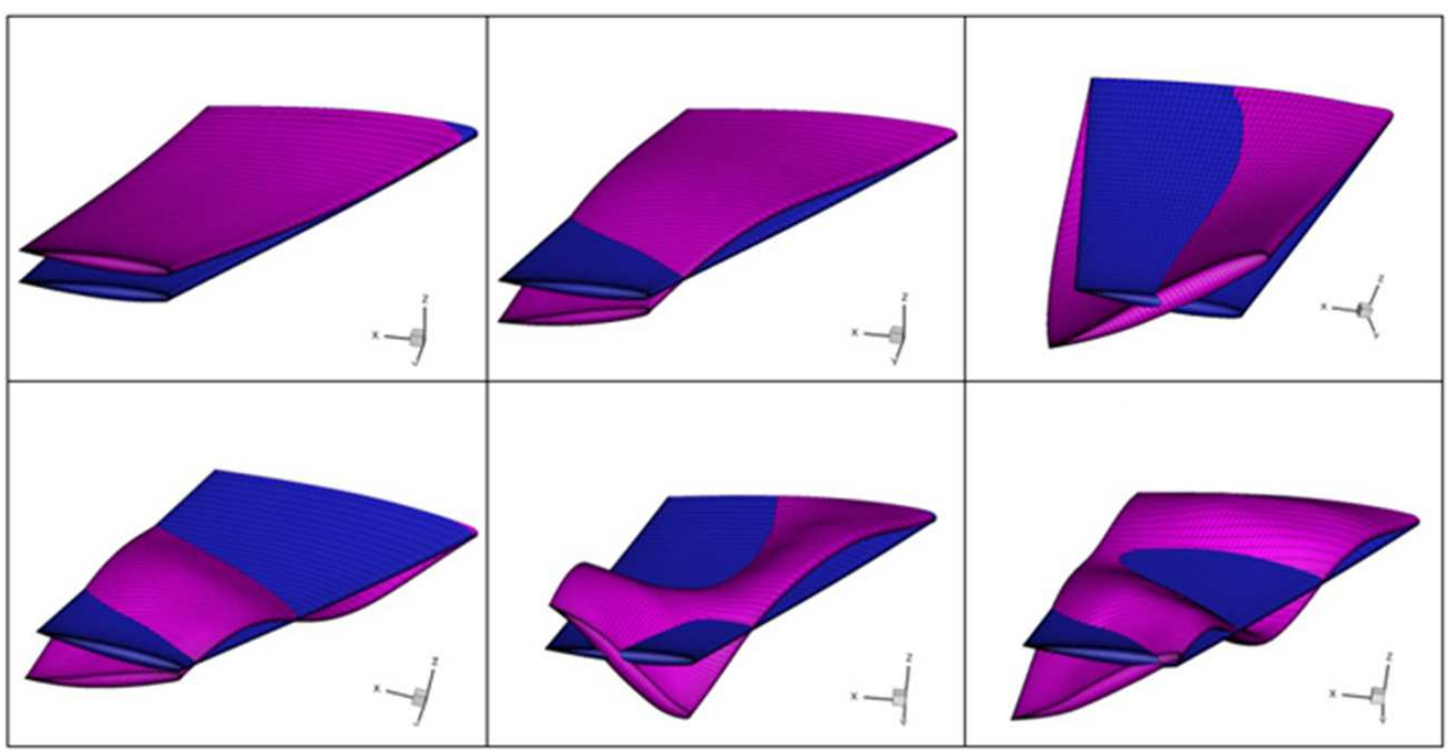

Fig. 3 First six structural mode shapes splined on the wetted surface.

reported in Fig. 4. In both direct and adjoint mode, two right hand sides are considered, i.e. one for each design parameter for the direct mode, and one for each objective function for the adjoint mode. Table 1 summarizes the total lift and drag coefficient derivative values, and allows a comparison 
between finite differences, intrusive approach and non-intrusive approach. Gradients obtained with finite differences and those obtained with the tangent approach are very close, which proves that the direct system has been correctly implemented. Moreover, the duality between tangent and adjoint approach is numerically preserved up to 6 significant digits.
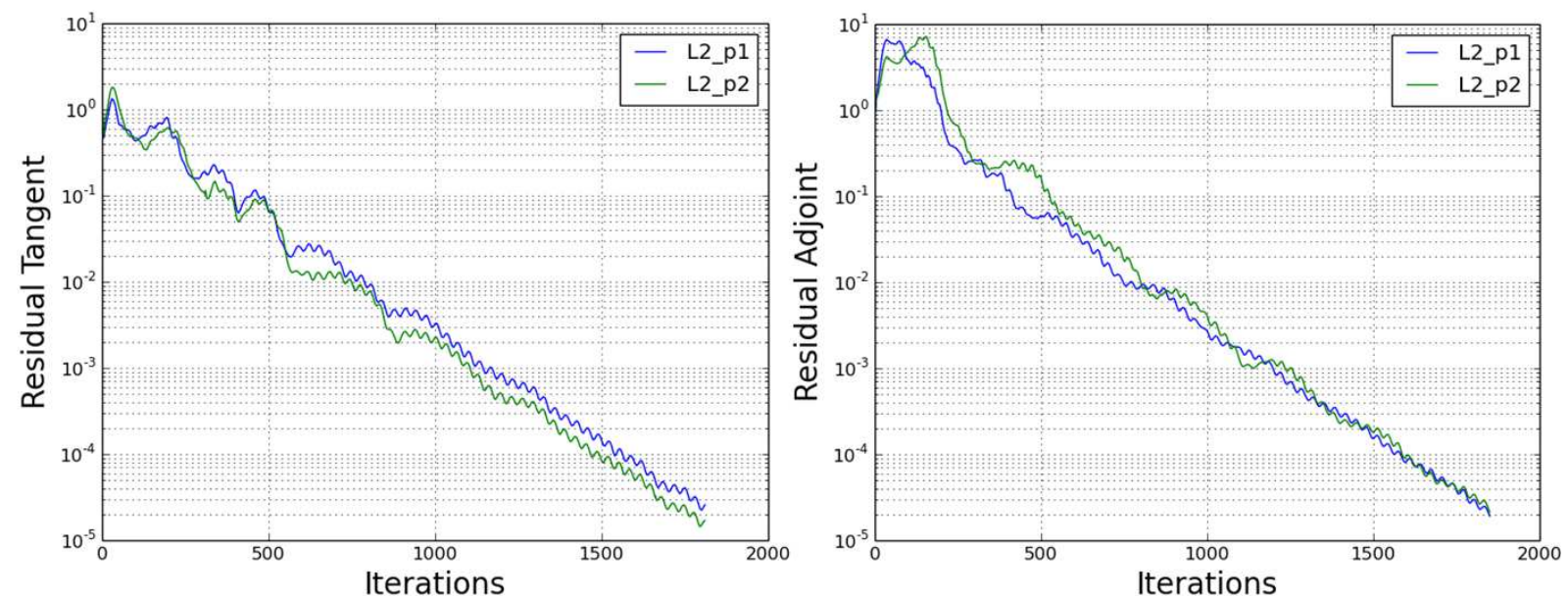

Fig. 4 Coupled-system sensitivity analysis. Convergence of the density residual for the coupled-tangent system with two parameters (left) and for the coupled-adjoint system with two functions (right).

Table 1 Gradients for lift and drag coefficients with respect to wing skin and spar thickness

\begin{tabular}{|c|c|c|c|c|c|c|}
\hline & & \multirow[b]{2}{*}{ FD } & \multicolumn{2}{|c|}{ Intrusive } & \multicolumn{2}{|c|}{ Non-Intrusive } \\
\hline & & & Direct & Adjoint & $\boldsymbol{\Phi}, \partial \boldsymbol{\Phi} / \partial p$ & $\Phi$ \\
\hline \multirow{2}{*}{$d C_{L} / d p$} & $p_{1}$ & $+1.61837 \times 10^{-2}$ & $+1.61901 \times 10^{-2}$ & $+1.61901 \times 10^{-2}$ & $+1.41747 \times 10^{-2}$ & $+1.83039 \times 10^{-2}$ \\
\hline & $p_{2}$ & $-5.63572 \times 10^{-3}$ & $-5.62821 \times 10^{-3}$ & $-5.62821 \times 10^{-3}$ & $-5.14122 \times 10^{-3}$ & $-6.86650 \times 10^{-3}$ \\
\hline \multirow{2}{*}{$d C_{D} / d p$} & $p_{1}$ & $+8.81343 \times 10^{-4}$ & $+8.81777 \times 10^{-4}$ & $+8.81777 \times 10^{-4}$ & $+7.89735 \times 10^{-4}$ & $+9.77248 \times 10^{-4}$ \\
\hline & $p_{2}$ & $-2.29081 \times 10^{-4}$ & $-2.28703 \times 10^{-4}$ & $-2.28703 \times 10^{-4}$ & $-2.04991 \times 10^{-4}$ & $-3.01135 \times 10^{-4}$ \\
\hline
\end{tabular}

These results validate the implementation of the direct and adjoint method into els $\boldsymbol{A}$ to compute gradients of aeroelastic functions of interest with respect to structural design variables. Finally, the non-intrusive approach with and without the eigenvector derivatives contribution $(\partial \mathbf{\Phi} / \partial p)$ gives satisfactory results, since the sign and the order of magnitude are always well predicted compared to the other approaches. We recall that without this contribution, the method is independent of 
the number of design variables, reducing dramatically the computational cost of the gradients.

For the particular case of aeroelastic coefficient derivatives with respect to structural parameters, there are usually few objective functions but many design variables. Therefore, the adjoint method is the preferred approach, since it requires one resolution of the adjoint system in Eq. 20 per objective function.

Now considering the total derivatives of the structural loads (i.e. aerodynamic load derivatives transferred on force nodes), the computational cost of the adjoint approach is indexed to the number of individual force components. Usually, a realistic structural loading consists of up to several hundred individual force components. This is comparable to the typical number of design parameters for a realistic optimization process. In this context, neither the tangent or adjoint method really stands out. However, computing the gradients of structural loads using the non-intrusive approach without the eigenvector derivatives contribution requires significantly lower computational cost as compared to the intrusive approaches. Again, in this case, aerostructural gradients only cost one rigid linearized gradient computation per mode shape.

Figure 5 highlights this discussion. On this plot, the full non-intrusive approach takes into account the eigenvector derivatives contribution, while its light version does not. Let $n_{f}$ be the number of individual force components, and $n_{\Phi}$ the number of modes retained in the modal basis $\left(n_{f} \gg n_{\Phi}\right)$. In the full version, the non-intrusive method has a lower slope than the tangent intrusive approach since only rigid linearized gradient computations are required to reconstruct the total gradient. In our case, the cost of one rigid linearized gradient computation is $30 \%$ lower than one aeroelastic linearized gradient computation. The choice of the gradient computation method is therefore driven by the number of retained modes (usually few), design variables, and individual force components. Finally, using the light non-intrusive approach will be in most cases the cheapest choice, but also the less accurate.

In order to assess the accuracy of the non-intrusive approach, total derivatives of the structural loads are computed with the tangent approach and compared to the non-intrusive approach (with and without eigenvector derivatives). To have a representative example, gradients of transverse forces with respect to $p_{1}$ and $p_{2}$ are considered. For sake of clarity, normalized gradients are plotted 


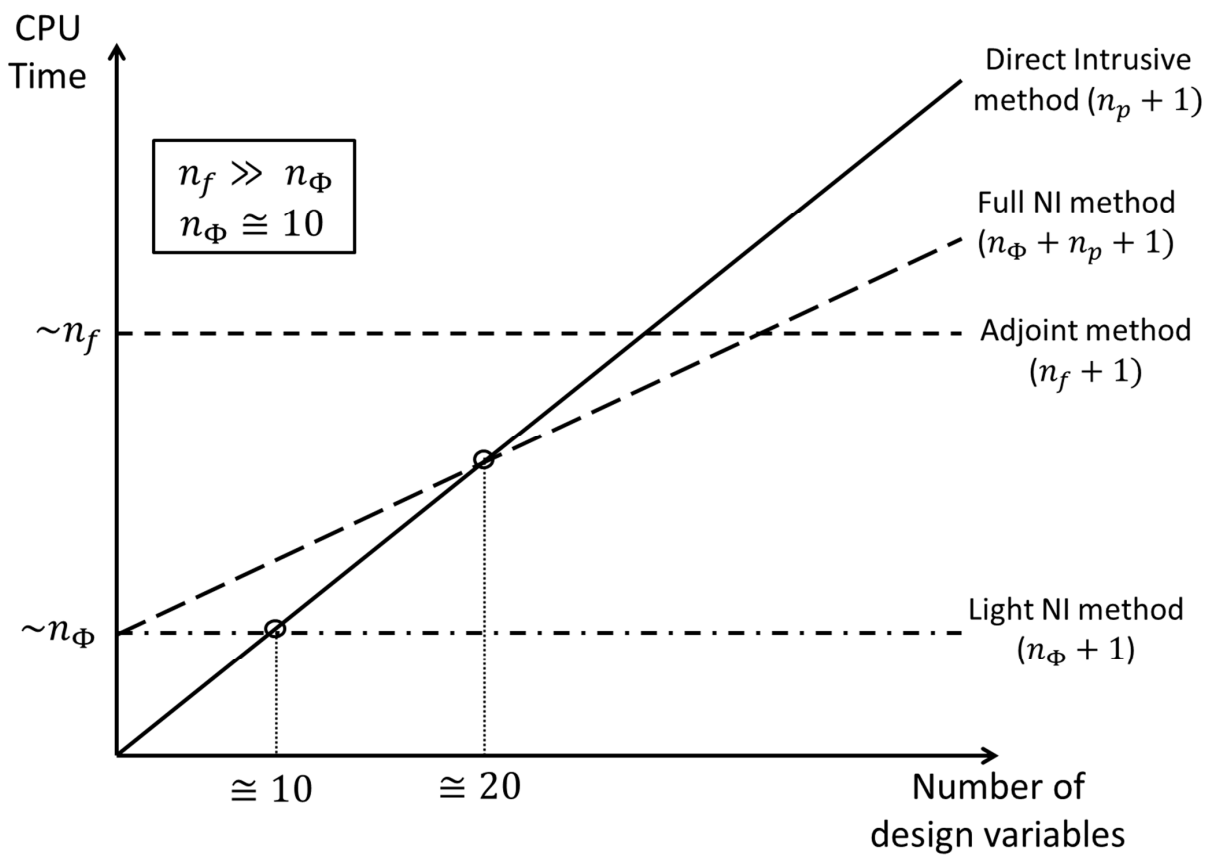

Fig. 5 Synthesis of the computational cost of the different gradient computation methods.

on nodes belonging to the front spar only (see Figs. 6-7). First of all, results for intrusive and nonintrusive approach qualitatively match pretty well. Taking the intrusive approach as reference, it is observed that the non-intrusive approach is always predictive, even without eigenvector derivatives contribution. However, for some nodes close to the wing root, gradients of transverse force with respect to $p_{2}$ computed without eigenvector derivatives are wrong. This points out the relevancy of adding the eigenvector derivatives contribution for some design variables. In [38], the authors propose a criterion that measures the amount of relevant information contained in the eigenvector derivatives compared to the information contained in the modal basis. It reveals whether a specific parameter requires the addition of eigenvector derivatives in the load sensitivity reconstruction. Nevertheless, it can be observed that these discrepancies occur mainly for low-valued gradients, which do not significantly affect gradient-based optimization since the optimizer's path is mostly driven by high-valued sensitivities. 


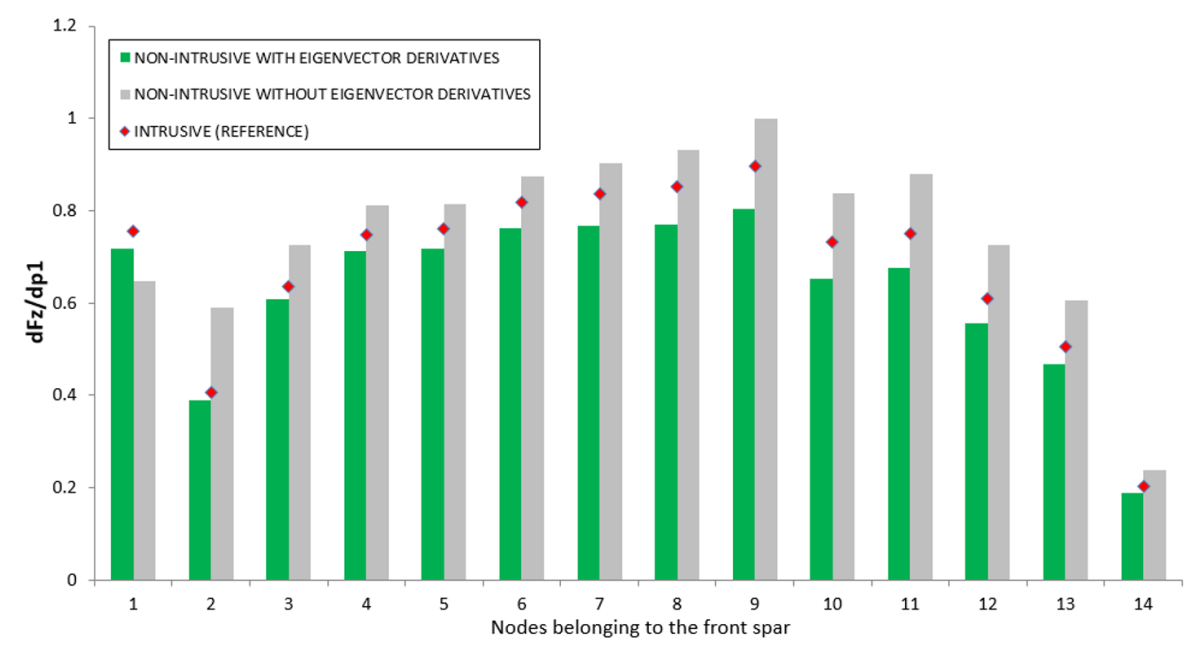

Fig. 6 Gradients of transverse forces with respect to $p_{1}$, computed with the non-intrusive approach (green and grey bars). Reference tangent approach values (red markers) are provided.

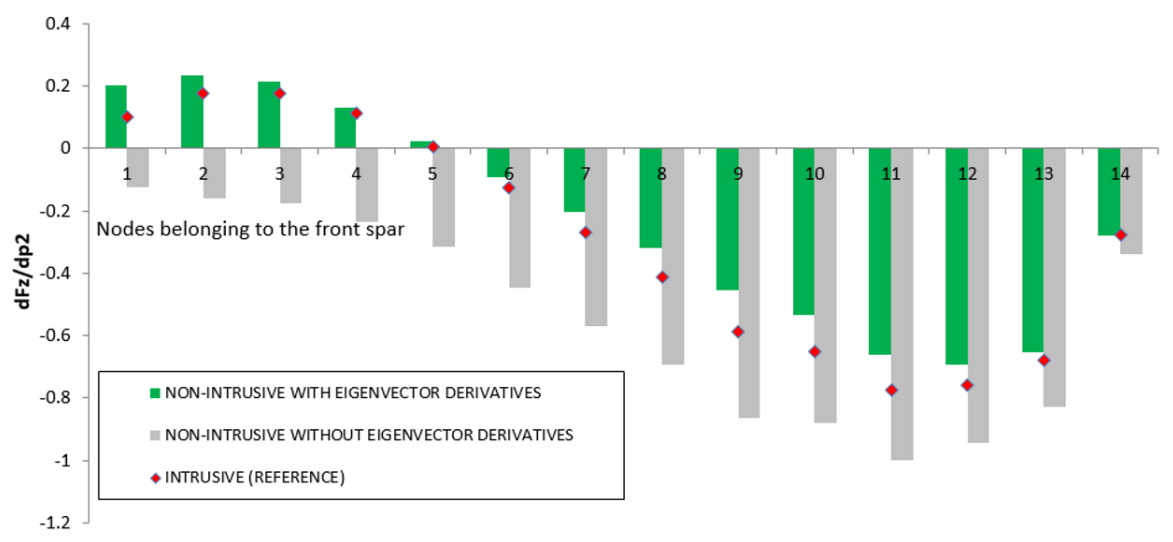

Fig. 7 Gradients of transverse forces with respect to $p_{2}$, computed with the non-intrusive approach (green and grey bars). Reference tangent approach values (red markers) are provided.

\section{Structural sizing of the CRM wing using high-fidelity aerostructural gradients}

\section{A. Force approximation method}

High-fidelity structural sizing requires derivatives of structural responses (typically constraints) with respect to structural parameters. These derivatives are costly, even when computed with the adjoint method since it requires as many resolution of the adjoint system as the total number of constraints in the whole structure. In an attempt to reduce the size of the set of admissible constraints, thus the number of gradients to compute, some authors have proposed to use a constraint aggregation technique in conjunction with the adjoint method. As already mentioned in the introduction, 
this approach needs to be tuned appropriately.

Approximating constraint responses is not easy, since they may exhibit an irregular distribution in the structure. In contrast, the structural loads are easier to approximate due to their smoother distribution. It is therefore proposed to use the force approximation method [39] as an alternative to a constraint aggregation approach. Assuming a general linear elastic behavior, the relationship between stresses and strains would be linear of the form $\sigma=\mathbf{H}\left(\epsilon-\epsilon_{0}\right)+\sigma_{0}$, where $\mathbf{H}$ denotes the elasticity matrix and $\epsilon_{0}$ and $\sigma_{0}$ the initial strain and stress fields. The deformation field is obtained from the nodal displacements through the usual relation $\epsilon=\mathbf{S N U}(\mathbf{p})=\mathbf{B U}(\mathbf{p})$, $\mathbf{S}$ being a linear differential operator and $\mathbf{N}$ the matrix of shape functions. Direct differentiation of $\sigma$ with respect to a scalar structural parameter $p$ yields:

$$
\frac{d \sigma}{d p}=\frac{\partial \sigma}{\partial \mathbf{U}} \frac{d \mathbf{U}}{d p}=\mathbf{H B} \frac{d \mathbf{U}}{d p}
$$

Deriving the static equation $\mathbf{K U}=\mathbf{Q}_{s}$ with respect to $p$ gives the expression of the structural displacement derivatives:

$$
\frac{d \mathbf{U}}{d p}=-\mathbf{K}^{-1} \frac{\partial \mathbf{K}}{\partial p} \mathbf{U}+\mathbf{K}^{-1} \frac{d \mathbf{Q}_{s}}{d p}
$$

Equation 39 becomes:

$$
\frac{d \sigma}{d p}=\mathbf{H B K}^{-1}\left(-\frac{\partial \mathbf{K}}{\partial p} \mathbf{U}+\frac{d \mathbf{Q}_{s}}{d p}\right)=\frac{d \sigma_{U}}{d p}+\frac{d \sigma_{Q}}{d p}
$$

where $d \sigma_{U} / d p$ is a constraint response under a standard static pseudo-load $\mathbf{F}_{U}=-(\partial \mathbf{K} / \partial p) \mathbf{U}$, and $d \sigma_{Q} / d p$ is a constraint response under the pseudo-load $\mathbf{F}_{Q}=d \mathbf{Q}_{s} / d p$. Therefore, for each static load $\mathbf{Q}_{s}$, a companion load $d \mathbf{Q}_{s} / d p$ must be defined. With this strategy, the total gradient of a constraint response can be recombined from two separate standard static analyses. Then, by linearity assumption, the two contributions in the right-hand side of Eq. 41 can be summed and the total gradient of the constraints is directly obtained, leaving the computational burden to the structural solver.

\section{B. Aeroelastic configuration of the Common Research Model (CRM)}

The aerodynamic skin of the selected wing/body/HTP (WBH) configuration is plotted in Fig. 8. For all subsequent results, a RANS fluid model is used with an upwind Roe scheme and a MUSCL 
interpolation associated to a Van Albada limiting function. The Spalart-Allmaras one-equation turbulence model is selected. In order to get a lift coefficient of 0.5 for a Mach number of 0.85 at $35000 \mathrm{ft}$, an angle of attack of $2.127^{\circ}$ is applied.

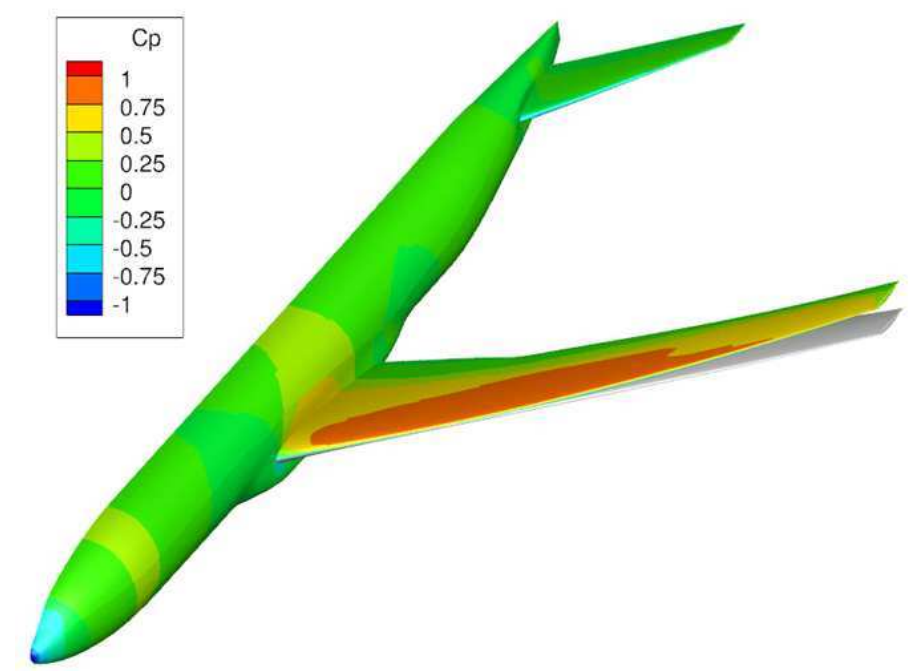

Fig. $8 \mathrm{Jig}$ and flight shape superimposed

Several finite element models of the CRM wing are available on the NASA CRM website. All these models correspond to the flight shape of the CRM. The model "V14" exhibits an expected static behavior, with a realistic vertical displacement of $2.15 \mathrm{~m}$ at the wing tip in nominal cruise conditions. It is a simple finite element model of the wing box and centre box with spars, skins, ribs, implicit stiffeners and a constant wing skin thickness of $8.89 \mathrm{~mm}$ (see Fig. 9). This FEM has 28092 degrees of freedom.

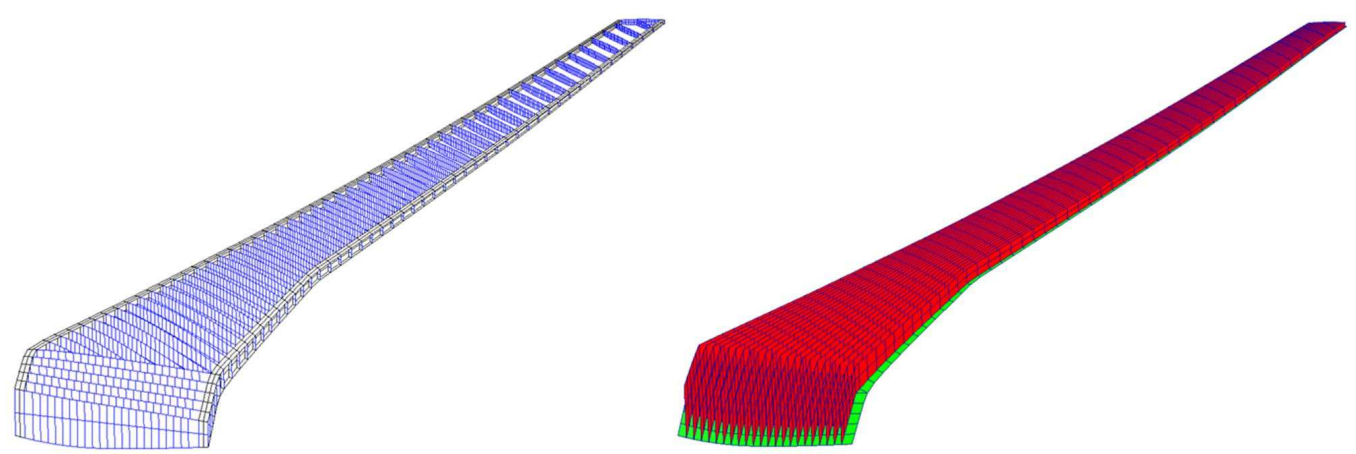

Fig. 9 Left: spars and ribs, right: lower skin and stringers.

This model can be considered as a good starting point for a structural sizing process. The 
provided CRM configuration corresponds to the $1 \mathrm{~g}$ cruise shape. In order to find the corresponding jig shape, an inverse procedure has been set up and presented in [38]. To correctly predict the structural displacement due to gravity, a realistic distribution of concentrated masses representing the fuel in the wing is added, along with concentrated masses for the engine, pylon and landing gear. The mass breakdown then corresponds to a realistic maximum take-off weight configuration.

\section{Proposed strategy for an efficient structural sizing}

Given a FEM of the CRM wing and the associated fluid mesh, our objective is to solve a mass minimization problem under several load cases and constraints. This optimization problem can be formulated as:

$$
\text { Minimize } M(\mathbf{p}) \text { such that: }\left\{\begin{array}{l}
\boldsymbol{\sigma}_{V M}(\mathbf{p}) \leq \boldsymbol{\sigma}_{\text {max }} \\
\mathbf{p}_{\text {min }} \leq \mathbf{p} \leq \mathbf{p}_{\text {max }}
\end{array}\right.
$$

where $M$ is the structural mass, and $\boldsymbol{\sigma}_{V M}$ are the Von Mises stresses. Figure 8 presents the $1 \mathrm{~g}$ flight shape colored by pressure coefficients, and the corresponding jig shape. Two critical load cases are considered in the structural optimization: $\mathrm{a}+2.5 \mathrm{~g}$ pull-up and $\mathrm{a}-1 \mathrm{~g}$ descent. The $+2.5 \mathrm{~g}$ and $-1 \mathrm{~g}$ load cases are generated by simply applying a multiplicative coefficient to the $1 \mathrm{~g}$ aeroelastic pressure distribution. This method is not an industrial practice, since it generates a conservative estimate of the loads that could prevent from finding the best design. However, this assumption is used here to obtain loads in a faster way.

The structure is assumed to be manufactured from Aluminum 2024. The corresponding limit Von Mises stress is $\boldsymbol{\sigma}_{\max }=260 . \mathrm{MPa}$. The ultimate loads are derived using a safety factor of 1.5. We define Von Mises stress constraints in all the 780 shell elements of the skins. The set of structural parameters $\mathbf{p}$ is defined as the thickness of 46 structural groups depicted in Fig. 10 and controlling the lower and upper skins.

The optimization process presented in Fig. 11 relies on the aerodynamic software els $\boldsymbol{A}$ for the computation of aeroelastic loads and associated gradients, and on the MSC NASTRAN software for the structural analysis and optimization part. The process has two levels of convergence: one 


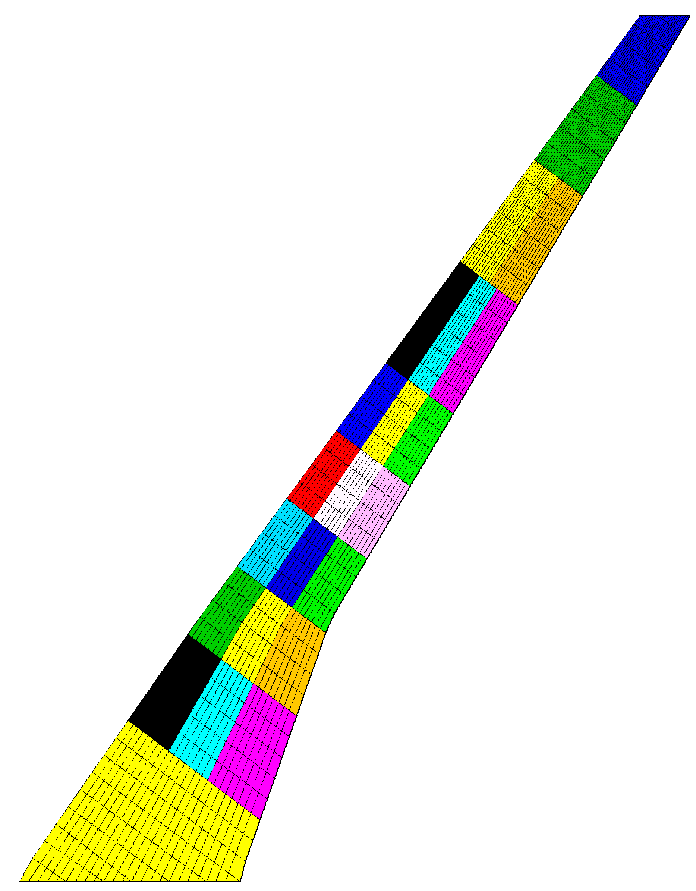

Fig. 10 Definition of the 46 structural design parameters (23 in each skin).

at the outer level on the load cases and one at the inner optimizer level on physical properties and design objective function. The structural optimization process (step 1) solves Eq. 42 for a given set of external non-linear aeroelastic loads. Then, the flexibility matrix $\mathbf{S}$ of the sized structural model and the structural displacements due to gravity are automatically derived in order to perform the adequate high-fidelity static aeroelastic and sensitivity analyses at the current iteration (step 2). Once the new flexible non-linear loads and associated sensitivities are obtained, they are transferred to the structural grid and a new optimization process is performed. This organization is very flexible as it does not require any modification to the core of the structural solver.

Figure 11 presents different optimization processes to solve problem 42, according to the selected strategy to compute the loads. If they derive from Doublet Lattice Method (DLM) only (first case), then the optimization strategy merely relies on standard structural solver capabilities.

The second case named "DLM+Correction" corresponds to the DLM prediction corrected by data from rigid CFD, thus requiring off-line $\boldsymbol{e l s} \boldsymbol{A}$ aerodynamic computations. This corresponds to the standard industrial practice for loads prediction. The third case is based on an external CFDbased Aeroelastic Analysis (A.A.) to estimate the loads. These aerodynamic loads are frozen during 
the internal optimization loop. Finally, the last case (Aeroelastic + Sensitivity Analysis) extends the previous one with the contribution of high-fidelity load sensitivities with respect to structural design variables. The structural optimizer then benefits from the additional contribution of these load sensitivities using the first order force approximation method.

In the first two cases, a strong coupling exists between the aerodynamic and the structural discipline, but the fidelity of the fluid model is limited. For the last two cases, of higher fidelity, there is a weak coupling between these two disciplines, since the loads and their sensitivities are not updated at each re-analysis of the FEM.

A strategy based on a strong coupling and high-fidelity models would involve the merge between the re-analysis loop and the external loads loop. Consequently, for each re-analysis asked by the structural optimizer, els $\boldsymbol{A}$ would be invoked to compute the loads and their sensitivities for the current set of structural parameters and to provide them to the structural optimizer. In Fig. 11, this means to replace the 20 re-analysis per external iteration by only one re-analysis per external iteration. However, this strategy is expected to be of higher computational cost.

As explained in section IV, the non-intrusive approach provides an efficient way to access gradients of loads. However, for cross-gradients like aerodynamic coefficient derivatives with respect to

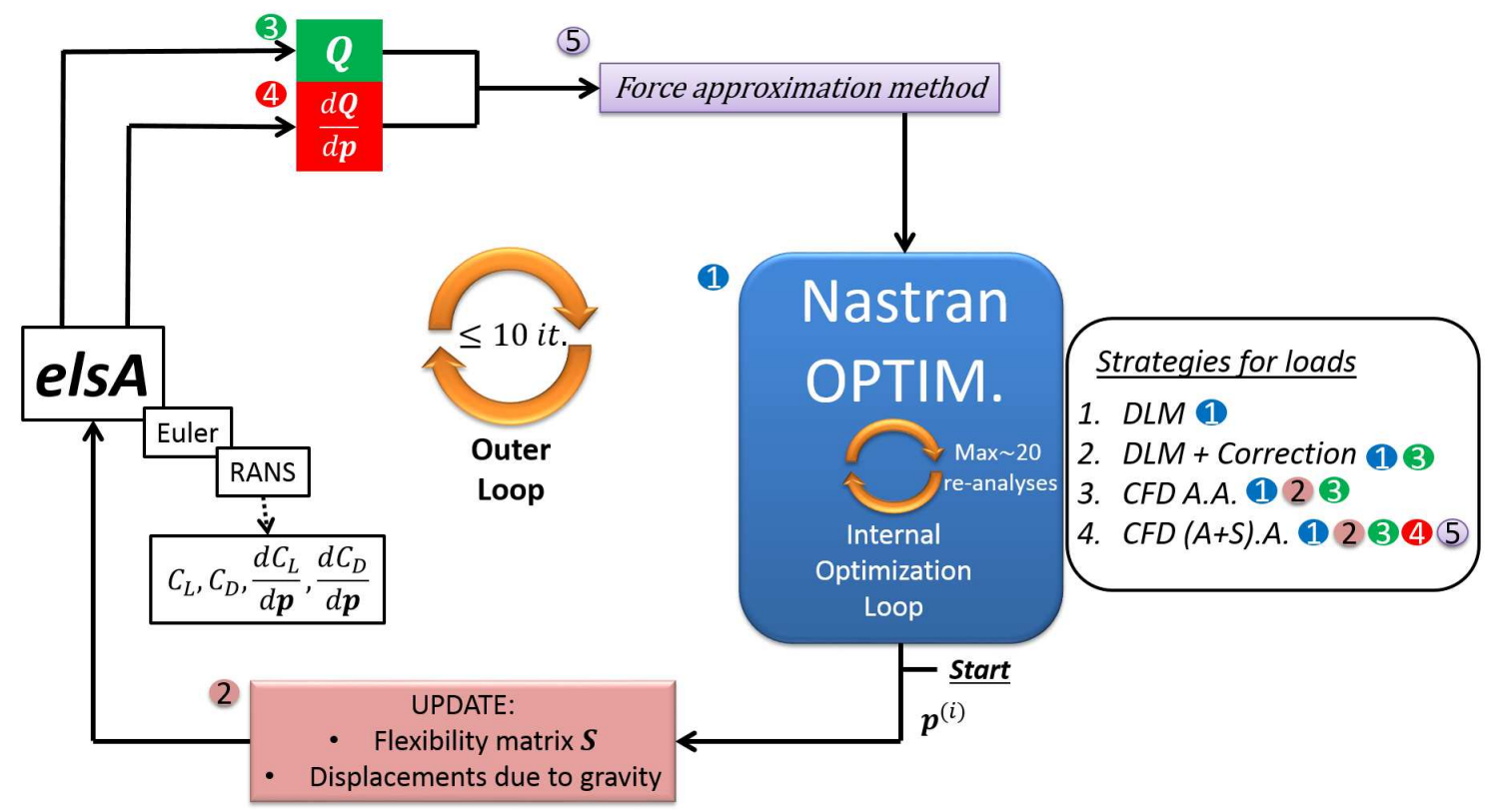

Fig. 11 Structural optimization workflow. 
structural parameters, the adjoint method is preferable. With both capabilities readily available, an aerodynamic performance criterion can be considered during the sizing process, particularly by taking into account $d C_{D} / d \mathbf{p}$ or $d C_{L} / d \mathbf{p}$.

\section{Results}

In this section, we present the optimization results obtained by applying the process described above. The strategy chosen is based on a weak coupling, i.e. the internal optimizer repeatedly asks for structural analyses until convergence but using a first order approximation of the highfidelity aeroelastic loads. At each external iteration, the loads and their sensitivities computed with the intrusive or non-intrusive approach are updated with high-fidelity analyses. This organization corresponds to the fourth case in Fig. 11. This strategy is similar to that presented in [42]. For information, the optimization method used in the NASTRAN SOL 200 is the Modified Method of Feasible Directions (MMFD, [43]). In both cases, the optimal design satisfies the constraints by increasing the structural mass. Starting with an initial skin mass of $4649.3 \mathrm{~kg}$ corresponding to a uniform thickness distribution of $8.89 \mathrm{~mm}$, the optimization process ends up after 4 external iterations with a mass of $4831.8 \mathrm{~kg}$ for the intrusive approach, and of $4845.1 \mathrm{~kg}$ for the non-intrusive one. The discrepancy between these optimal values is less than $0.3 \%$. 


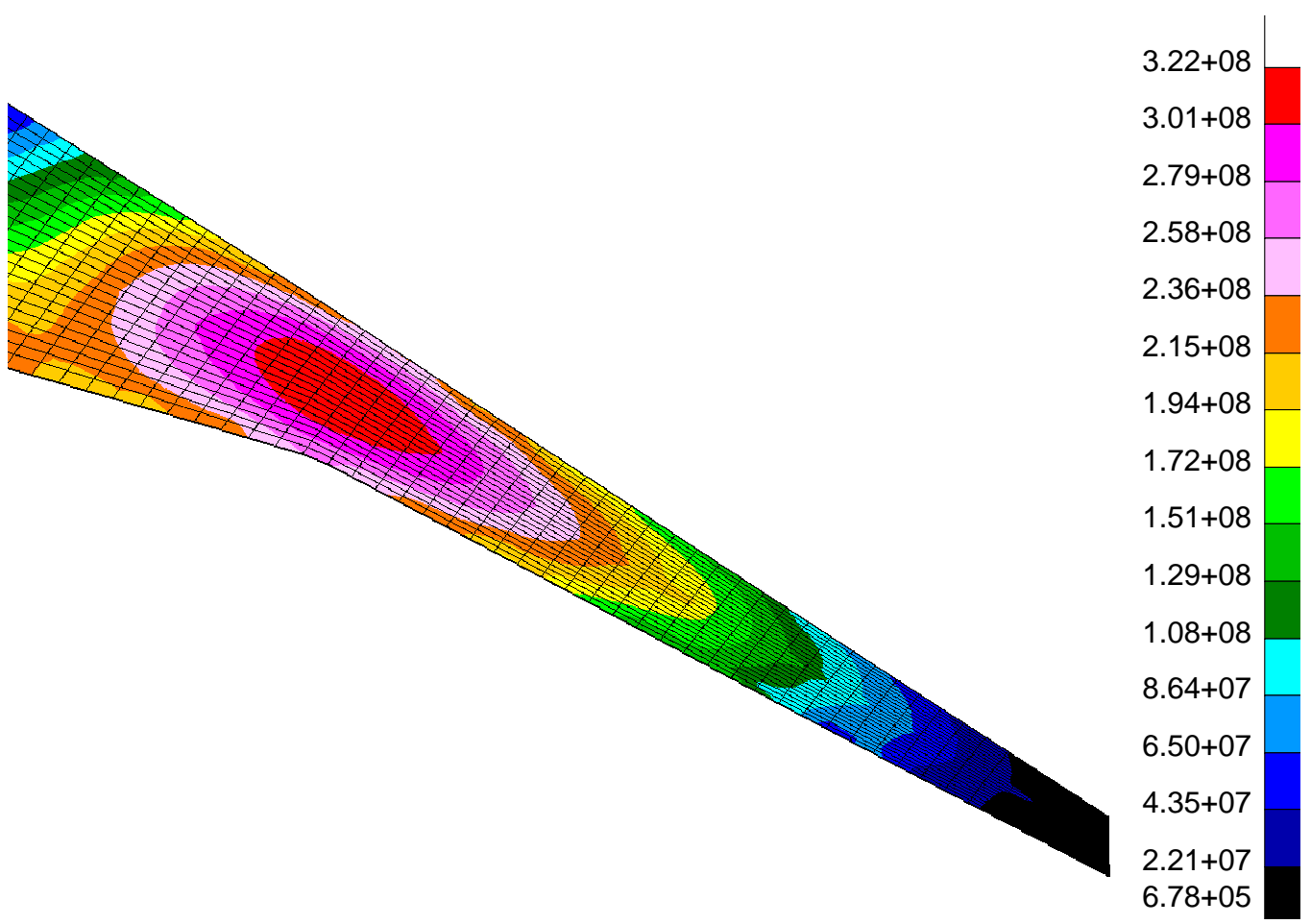

Fig. 12 Initial Von Mises stress distribution on the upper skin under $2.5 \mathrm{~g}$ load case.

Figure 12 presents the initial Von Mises stress distribution on the upper skin under the critical $2.5 \mathrm{~g}$ load case. The initial configuration is infeasible with 47 active constraints (i.e. constraint values close to the true constraint boundary) and 379 violated. 


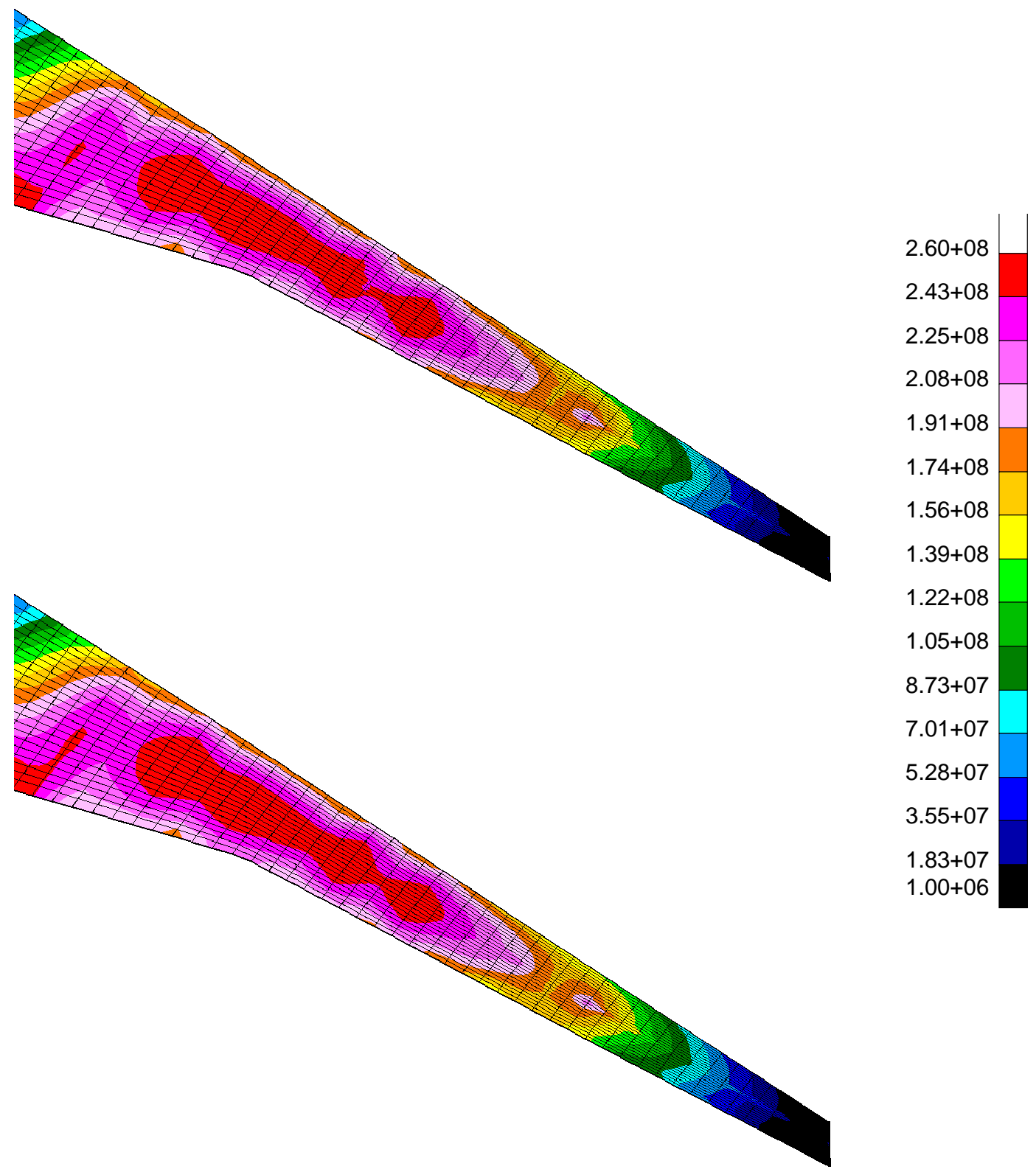

Fig. 13 Comparison of final Von Mises stress distribution on the upper skin sized using the intrusive formulation (top) and the non-intrusive formulation (bottom).

Figure 13 compares the final stress distributions obtained with the two gradient computation approaches under the $2.5 \mathrm{~g}$ load case. Both approaches lead to almost identical stress distributions, and end up with a feasible design (no violated constraints). There are respectively 154 and 181 active constraints for the intrusive and non-intrusive approach.

Similarly, Fig. 14 compares the final thickness distributions obtained with the two gradient 
computation approaches. Again, both approaches present very similar thickness distributions.
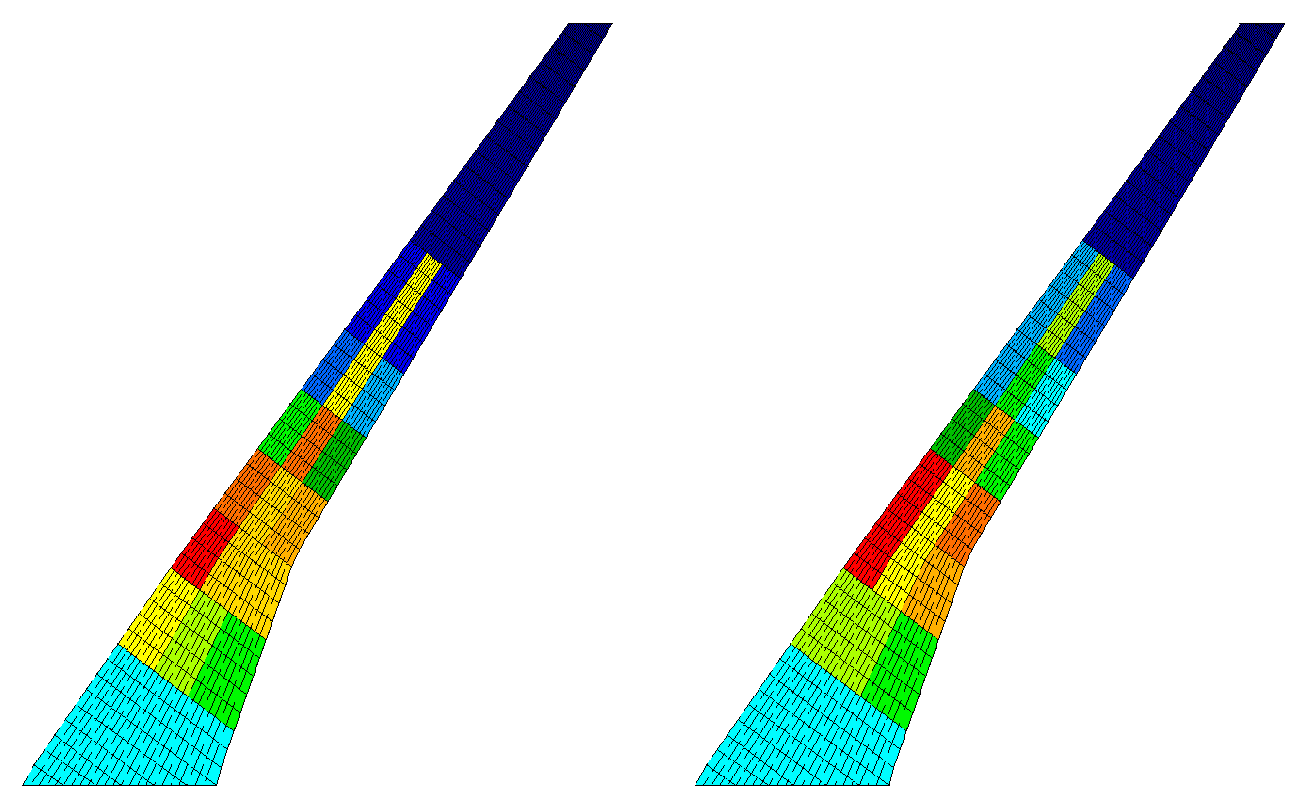

1.52-02

Fig. 14 Final thickness distribution on the upper skin using the intrusive approach (left) and the non-intrusive approach (right).

The new designs obtained with both gradient computation approaches are very close. This is the demonstration that the non-intrusive approach is accurate enough to converge a realistic design process such as a mass minimization problem. This approach is therefore a credible alternative to the classical intrusive formulations.

In this optimization problem, 46 design variables are defined, and the total number of active and violated constraints is always greater than 154. In such case, a constraint aggregation method should be used to keep the efficiency of the adjoint approach. However, using the force approximation method associated with the intrusive or non-intrusive approach avoids the need for such an aggregation. In this case, the CPU time is indexed at most on the number of design variables, and no approximation on the constraint responses has been introduced. In practice, we recall that the cost of one rigid linearized gradient computation is $30 \%$ lower than one aeroelastic linearized gradient computation (see Fig. 5). A simple calculation indicates that for 20 design variables or more, the total cost favors the full non-intrusive approach. In the present case, using the non-intrusive approach requires less computational time than using the direct one. 


\section{Conclusions}

In this paper two approaches are presented to compute high-fidelity aerostructural gradients. The first one is based on the classical intrusive tangent and adjoint approaches, and the second one is an uncoupled modal-based non-intrusive method benefiting from the existing linearized aerodynamic module dedicated to gradient computation. Using both approaches, total derivatives of structural loads along with lift and drag coefficients with respect to structural design parameters are computed on the ONERA M6 Wing test-case.

Gradients obtained with the tangent approach have been taken as reference since the accuracy has been validated with finite difference results. The very low discrepancies between tangent and adjoint gradient values demonstrate that the duality between the two methods is numerically preserved. These results validate the implementation of the tangent and adjoint method into els $\boldsymbol{A}$.

The non-intrusive reconstruction gives satisfactory results compared to the other approaches. The strength of this approach relies on its relatively low computational cost, but cannot overcome the adjoint method for the particular case of aerodynamic coefficient derivatives. Still, it represents a very promising alternative to compute gradients of structural loads for structural sizing. Usually, for this purpose, constraint aggregation technique is used in conjunction with the adjoint method. However, limiting the number of stress responses in the sizing process may oversimplify the problem formulation, leading to poor designs. In contrast, considering a limited number of structural loads can still lead to a realistic load path and then to a predictive set of stress responses. This fact obviously favors the direct approach which in this specific case exhibits a comparable cost to the adjoint approach. We red recall that computing the gradients of structural loads using the nonintrusive approach requires only rigid linearized computations. This feature is essential for those who do not have access to strongly coupled aerostructural tangent or adjoint solver. Finally, uncoupled approaches are very interesting for MDO teams autonomy, which is a determining advantage in an industrial optimization process.

As a demonstration, a mass minimization problem is set up on the CRM wing. To this end, an efficient structural sizing using high-fidelity aerostructural gradients is proposed. This strategy is based on the force approximation method, considered as a competitive alternative to a constraint 
aggregation approach. Indeed, due to their smoother distribution, the structural loads are easier to approximate than the stresses. Several optimization processes are envisaged, according to the strategy selected to compute the loads. Results are presented in the context of a weak coupling strategy, where the loads and their sensitivities are updated with high-fidelity analyses at each outer iteration. Very close results are obtained using intrusive or non-intrusive approach. The potential of the non-intrusive approach is therefore demonstrated, and can be now considered as a credible alternative to the classical intrusive formulations.

\section{Future work}

Improvements on the non-intrusive approach with respect to the methodology itself and the associated numerical cost are currently under active investigation and will be detailed in a future paper. More specifically, we will propose an enhanced hybrid tangent/adjoint formulation that will completely remove the dependency of the non-intrusive approach to the number of design parameters thus dramatically reducing the numerical cost.

In this paper, the efficiency of the non-intrusive approach has been demonstrated on a design test-case limited to structural design variables only. However, we have shown in section III C that the method can be extended to take into account shape design variables affecting aerodynamic and structural grid coordinates at the same computional cost and is therefore expected to be equally efficient in a full aerostructural optimization. This approach is also easier to implement if an aerodynamic shape gradient computation module is already available as it then re-uses already available software bricks. This extension is currently being implemented and will be applied to a realistic aerostructural optimization in the near future.

\section{References}

[1] Adelman, H. M. and Haftka, R. T., "Sensitivity Analysis of Discrete Structural Systems," AIAA Journal, Vol. 24, No. 5, 1986, pp. 823-832, doi:10.2514/3.48671.

[2] Hicks, R. M. and Szelazek, C. A., "Airfoil Design by Numerical Optimization Using a Minicomputer," Tech. rep., NASA Technical Memorandum 78502, 1978. 
[3] Barthelemy, J.-F. M. and Bergen, F., "Shape Sensitivity Analysis of Wing Static Aeroelastic Characteristics," Tech. rep., NASA Technical Paper 2808, 1988.

[4] Shirk, M. H., Hertz, T. J., and Weisshaar, T. A., "Aeroelastic Tailoring-Theory, Practice, and Promise," Journal of Aircraft, Vol. 23, No. 1, 1986, pp. 6-18, doi:10.2514/3.45260.

[5] Grossman, B., Gurdal, Z., Strauch, G. J., Eppard, W. M., and Haftka, R. T., "Integrated Aerodynamic/Structural Design of a Sailplane Wing," Journal of Aircraft, Vol. 25, No. 9, 1988, pp. 855-860, doi: $10.2514 / 3.45670$.

[6] Chittick, I. R. and Martins, J. R. R. A., "An asymmetric suboptimization approach to aerostructural optimization," Optimization and Engineering, Vol. 10, No. 1, 2009, pp. 133-152, doi:10.1007/s11081-008-9046-2.

[7] Hutchison, M., Unger, E., Mason, W., Grossman, B., and Haftka, R., "Variable-Complexity Aerodynamic Optimization of an HSCT Wing using Structural Wing-Weight Equations," Journal of Aircraft, Vol. 31, No. 1, 1994, pp. 110-116, doi: $10.2514 / 3.46462$.

[8] McQuade, P. D., Eberhardt, S., and Livne, E., "CFD-Based Aerodynamic Approximation Concepts Optimization of a Two-Dimensional Scramjet Vehicle," Journal of Aircraft, Vol. 32, No. 2, 1995, pp. 262-269, doi: $10.2514 / 3.46711$.

[9] Knoll, D. and Keyes, D., "Jacobian-free Newton-Krylov methods: a survey of approaches and applications," Journal of Computational Physics, Vol. 193, No. 2, 2004, pp. 357-397, doi:10.1016/j.jcp.2003.08.010.

[10] Zhang, Z. J. and Zingg, D. W., "Efficient Monolithic Solution Algorithm for High-Fidelity Aerostructural Analysis and Optimization," AIAA Journal, Vol. 56, No. 3, 2018, pp. 1251-1265, doi:10.2514/1.J056163.

[11] Sobieszczanski-Sobieski, J., Bloebaum, C. L., and Hajela, P., "Sensitivity of Control-augmented Structure obtained by a System Decomposition Method," AIAA Journal, Vol. 29, No. 2, 1991, pp. 264-270, doi: $10.2514 / 3.10573$.

[12] Martins, J. R. R. A., Sturdza, P., and Alonso, J. J., "The Complex-Step Derivative Approximation," ACM Transactions on Mathematical Software, Vol. 29, No. 3, 2003, pp. 245-262, doi:10.1145/838250.838251.

[13] Sobieszczanski-Sobieski, J., "Sensitivity of Complex, Internally Coupled Systems," AIAA Journal, 
Vol. 28, No. 1, 1990, pp. 153-160,

doi: $10.2514 / 3.10366$.

[14] Maute, K., Nikbay, M., and Farhat, C., "Coupled Analytical Sensitivity Analysis and Optimization of Three-Dimensional Nonlinear Aeroelastic Systems," AIAA Journal, Vol. 39, No. 11, 2001, pp. 20512061 doi:10.2514/2.1227.

[15] Jameson, A., "Aerodynamic Design via Control Theory," Journal of Scientific Computing, Vol. 3, No. 3, 1988, pp. 233-260, doi:10.1007/BF01061285.

[16] Reuther, J., Jameson, A., Farmer, J., Martinelli, L., and Saunders, D., "Aerodynamic Shape Optimization of Complex Aircraft Configurations via an Adjoint Formulation," in "34th Aerospace Sciences Meeting and Exhibit, Aerospace Sciences Meetings," , 1996, doi:10.2514/6.1996-94.

[17] Maute, K., Nikbay, M., and Farhat, C., "Analytically Based Sensitivity Analysis and Optimization of Nonlinear Aeroelastic Systems," in "8th Symposium on Multidisciplinary Analysis and Optimization AIAA Paper 2000-4825,", 2000, doi: $10.2514 / 6.2000-4825$.

[18] Jameson, A., "Efficient Aerodynamic Shape Optimization," in "10th AIAA/ISSMO Multidisciplinary Analysis and Optimization Conference, Albany, New-York," , 2004, doi:10.2514/6.2004-4369.

[19] Gauger, N. R., "Adjoint Approaches in Aerodynamic Shape Optimization and MDO Context," in "European Conference on Computational Fluid Dynamics ECCOMAS CFD," , 2006.

[20] Salah El Din, I., Carrier, G., and Mouton, S., "Discrete Adjoint Method in elsA (part 2): Application to Aerodynamic Design Optimisation," in "7th ONERA-DLR Aerospace Symposium (ODAS), Toulouse," 2006.

[21] Mavriplis, D., "Discrete Adjoint-Based Approach for Optimization Problems on Three-Dimensional Unstructured Meshes," AIAA Journal, Vol. 45, No. 4, 2007, pp. 741-750, doi:10.2514/1.22743.

[22] Bücker, M., Corliss, G., Hovland, P., Naumann, U., and Norris, B., Automatic Differentiation: Applications, Theory, and Implementations, LNCSE. Springer, 2006.

[23] Hascoët, L., "Adjoints by Automatic Differentiation," in "Advanced Data Assimilation for Geosciences, Oxford University Press, 978-0-19-872384-4," , 2014. 
[24] Kenway, G. K. W., Kennedy, G. J., and Martins, J. R. R. A., "Scalable Parallel Approach for HighFidelity Steady-State Aeroelastic Analysis and Adjoint Derivative Computations," AIAA Journal, Vol. 52, No. 5, 2014, pp. 935-951, doi:10.2514/1.J052255.

[25] Sanchez, R. and Palacios, R., "Computing Derivatives in Nonlinear Aeroelasticity using Algorithm Differentiation," in "IFASD 2017, Como, Italy," , 2017.

[26] Marcelet, M., Peter, J., and Carrier, G., "Sensitivity Analysis of a Strongly Coupled System Using the Discrete Direct and Adjoint Approach," Revue Européenne de mécanique numérique, Vol. 17, 2008, pp. $1077-1106$ doi:10.3166/remn.17.1077-1106.

[27] Ghazlane, I., Carrier, G., and Dumont, A., "Aerostructural Adjoint Method for Flexible Wing Optimization," in "53rd AIAA/ASME/ASCE/AHS/ASC Structures, Structural Dynamics, and Materials Conference - AIAA Paper 2012-1924," , 2012, doi: $10.2514 / 6.2012-1924$.

[28] Viti, A., Druot, T., and Dumont, A., "Aero-Structural Approach Coupled with Direct Operative Cost Optimization for New Aircraft Concept in Preliminary Design," in "17th AIAA/ISSMO Multidisciplinary Analysis and Optimization Conference, AIAA Paper 2016-3512,”, 2016, doi:10.2514/6.2016-3512.

[29] Jameson, A., "Aerodynamic-Structural Design Studies of Low-Sweep Transonic Wings," Journal of Aircraft, Vol. 47, No. 2, 2010, pp. 505-514, doi:10.2514/1.42775.

[30] Brezillon, J., Ronzheimer, A., Haar, D., Abu-Zurayk, M., Lummer, M., Krüger, W., and Natterer, F. J., "Development and Application of Multi-Disciplinary Optimization Capabilities based on High-Fidelity Methods," in "53rd AIAA/ASME/ASCE/AHS/ASC Structures, Structural Dynamics and Materials Conference,", 2012.

[31] Blondeau, C., Achard, T., Girodroux-Lavigne, P., and Ohayon, R., "Recent Achievements towards Aero-Structure Gradient Computation using High-Fidelity CFD-CSM in the Onera elsA Software," in "International Forum on Aeroelasticity and Structural Dynamics, IFASD 2015, Saint Petersburg, Russia,", 2015.

[32] Girodroux-Lavigne, P., "Recent Navier-Stokes Aeroelastic Simulations using the elsA code for Aircraft Applications," in "International Forum on Aeroelasticity and Structural Dynamics, Stockholm, Sweden," , 2007. 
[33] Akgün, M. A., Haftka, R. T., Wu, K. C., Walsh, J. L., and Garcelon, J. H., "Efficient Structural Optimization for Multiple Load Cases Using Adjoint Sensitivities," AIAA Journal, Vol. 39, No. 3, 2001, pp. $511-516$, doi:10.2514/2.1336.

[34] Raspanti, C., Bandoni, J., and Biegler, L., "New Strategies for Flexibility Analysis and Design under Uncertainty," Computers $\&$ Chemical Engineering, Vol. 24, No. 9, 2000, pp. 2193 - 2209, doi:10.1016/S0098-1354(00)00591-3.

[35] Poon, N. M. K. and Martins, J. R. R. A., "An Adaptive Approach to Constraint Aggregation Using Adjoint Sensitivity Analysis," Structural and Multidisciplinary Optimization, Vol. 34, No. 1, 2007, pp. 61-73, doi:10.1007/s00158-006-0061-7.

[36] Abu-Zurayk, M., Ilić, C., Schuster, A., and Liepelt, R., "Effect of Gradient Approximations on AeroStructural Gradient-Based Wing Optimization," in "EUROGEN 2017, ECCOMAS Thematic Conference, Madrid, Spain,” , 2017.

[37] Lambe, A. B., Martins, J. R. R. A., and Kennedy, G. J., "An evaluation of constraint aggregation strategies for wing box mass minimization," Structural and Multidisciplinary Optimization, Vol. 55, No. 1, 2017, pp. 257-277, doi:10.1007/s00158-016-1495-1.

[38] Achard, T., Blondeau, C., and Ohayon, R., "An Uncoupled Approach to Compute Aero-Structure Gradients Using High-Fidelity CFD-CSM," in "17th AIAA/ISSMO Multidisciplinary Analysis and Optimization Conference, AIAA Paper 2016-4121,”, 2016, doi:10.2514/6.2016-4121.

[39] Vanderplaats, G. N. and Salajegheh, E., "New Approximation Method for Stress Constraints in Structural Synthesis," AIAA Journal, Vol. 27, No. 3, 1989, pp. 352-358, doi:10.2514/3.10119.

[40] Schmitt, V. and Charpin, F., "Pressure Distributions on the ONERA M6 Wing at Transonic Mach Numbers," Tech. rep., Experimental Data Base for Computer Program Assessment, AGARD Advisory Report AR-138, 1979.

[41] Vassberg, J. C., DeHann, M. A., Rivers, S. M., and Wahls, R. A., "Development of a Common Research Model for Applied CFD Validation Studies," AIAA Paper 2008-6919, doi:10.2514/6.2008-6919.

[42] Blondeau, C. and Salah El Din, I., "A Bi-Level High Fidelity Aero-Structural Integrated Design Method- 
ology - A Focus on the Structural Sizing Process," in "RAeS 3rd Aircraft Structural Design Conference, Delft, The Netherlands,", 2012.

[43] Vanderplaats, G. N., "An Efficient Feasible Directions Algorithm for Design Synthesis," AIAA Journal, Vol. 22, No. 11, 1984, pp. 1633-1640,

doi:10.2514/3.8829. 